\title{
Effects of Substitutional Dopants on the Photoresponse of a Polyoxotitanate Cluster
}

\author{
Junyi Hu ${ }^{\dagger}$, Lijie Zhan ${ }^{\dagger}$, Guanyun Zhang ${ }^{\dagger}$, Qun Zhang ${ }^{\ddagger}$, Lin Du ${ }^{\ddagger}$, Chen-Ho Tung ${ }^{\dagger}$, Yifeng Wang ${ }^{*}, \dagger$
}

${ }^{\dagger}$ Key Lab for Colloid and Interface Science of Ministry of Education, School of Chemistry and Chemical Engineering and ${ }^{\ddagger}$ Environment Research Institute, Shandong University, Ji 'Nan 250100, People’s Republic of China

\section{Table of contents}

1. Summary of syntheses

2. Single crystal X-ray crystallographic data

3. UV-vis titration of $\left\{\mathrm{Ti}_{11}\right\}$

4. Powder X-ray diffraction of $\left\{\mathrm{Ti}_{11} \mathrm{ML}\right\}$.

5. Fourier transform IR spectra

6. ${ }^{13} \mathrm{C}$ NMR spectra and ${ }^{1} \mathrm{H}$ NMR spectra

7. Band gap measurements

8. DFT calculations

9. UV-vis absorption spectra

10. The UV-vis titrations using $\left[\mathrm{FeCp}_{2}\right] \mathrm{BF}_{4}$

11. The ESR spectra and the simulated curves

12. The photocurrent measurements

13. Photoluminescence decay spectra 


\section{Summary of the syntheses}

Table S1. Syntheses and characterization technique of compounds

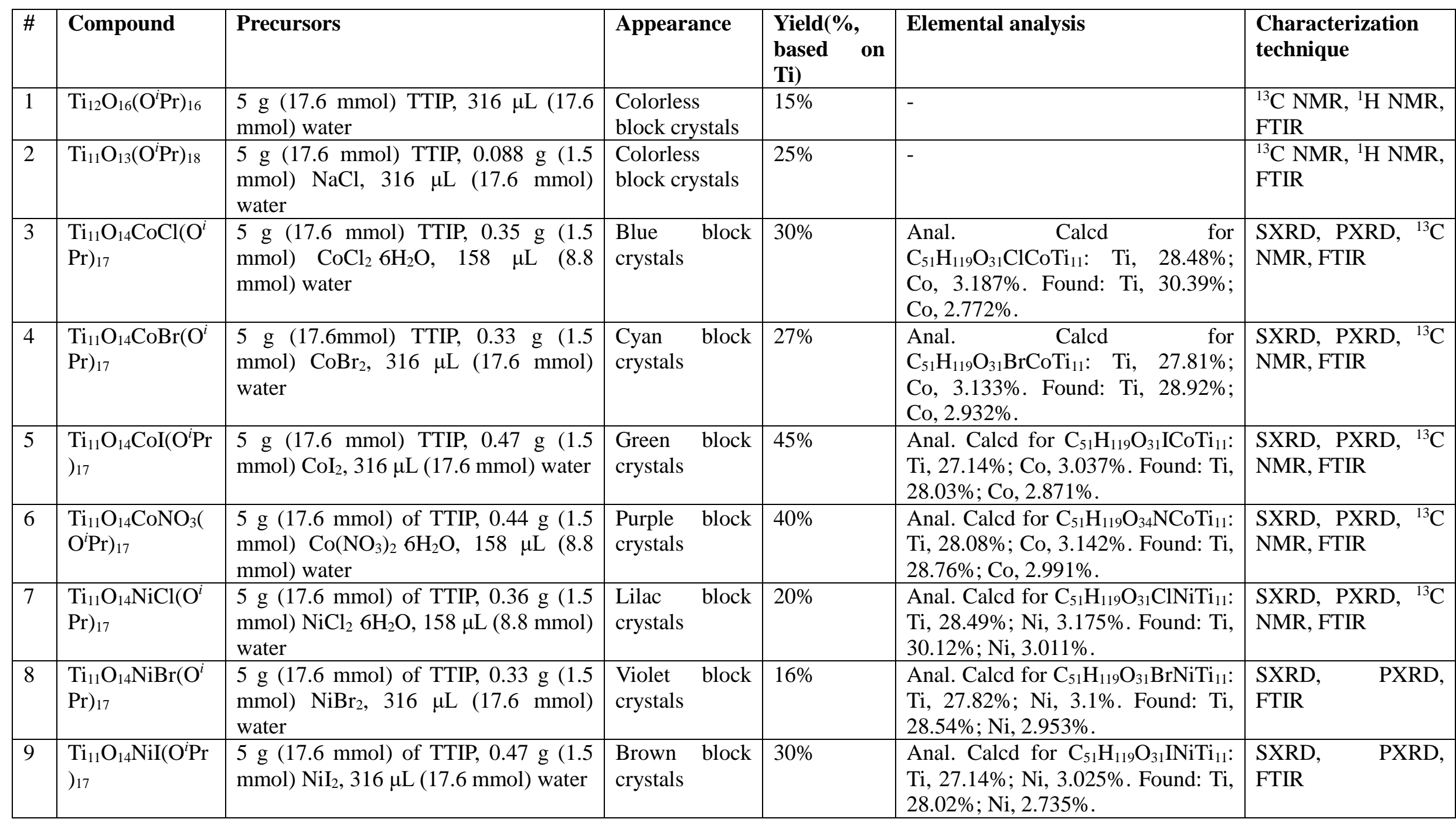




\begin{tabular}{|c|c|c|c|c|c|c|}
\hline 10 & $\begin{array}{l}\mathrm{Ti}_{11} \mathrm{O}_{14} \mathrm{MgCl}\left(\mathrm{O}^{i}\right. \\
\mathrm{Pr})_{17}\end{array}$ & $\begin{array}{l}5 \mathrm{~g}(17.6 \mathrm{mmol}) \text { of TTIP, } 0.3 \mathrm{~g}(1.5 \\
\text { mmol }) \mathrm{MgCl}_{2} \cdot 6 \mathrm{H}_{2} \mathrm{O}, 158 \mu \mathrm{L} \quad(8.8 \\
\text { mmol) water }\end{array}$ & $\begin{array}{l}\text { Colorless } \\
\text { block crystals }\end{array}$ & $10 \%$ & 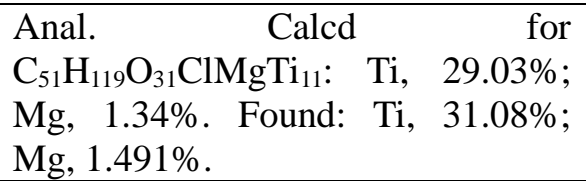 & $\begin{array}{l}\text { SXRD, PXRD, }{ }^{13} \mathrm{C} \\
\text { NMR, FTIR }\end{array}$ \\
\hline 11 & $\begin{array}{l}\mathrm{Ti}_{11} \mathrm{O}_{14} \mathrm{MgBr}\left(\mathrm{O}^{i}\right. \\
\mathrm{Pr})_{17}\end{array}$ & $\begin{array}{l}5 \mathrm{~g}(17.6 \mathrm{mmol}) \text { of TTIP, } 0.28 \mathrm{~g}(1.5 \\
\text { mmol }) \mathrm{MgBr}_{2}, 316 \mu \mathrm{L}(17.6 \mathrm{mmol}) \\
\text { water }\end{array}$ & $\begin{array}{l}\text { Colorless } \\
\text { block crystals }\end{array}$ & $16 \%$ & $\begin{array}{llr}\text { Anal. } & \text { Calcd } & \text { for } \\
\mathrm{C}_{51} \mathrm{H}_{119} \mathrm{O}_{31} \mathrm{BrMgTi}_{11}: & \mathrm{Ti}, & 28.33 \% \\
\mathrm{Mg}, 1.31 \% \text {. Found: } & \mathrm{Ti}, & 29.52 \% \\
\mathrm{Mg}, 1.432 \% . & & \end{array}$ & $\begin{array}{l}\text { SXRD, PXRD, } \\
\text { FTIR }\end{array}$ \\
\hline 12 & $\begin{array}{l}\mathrm{Ti}_{11} \mathrm{O}_{14} \mathrm{CaCl}\left(\mathrm{O}^{i}\right. \\
\mathrm{Pr})_{17}\left(\mathrm{HO}^{i} \mathrm{Pr}\right)\end{array}$ & $\begin{array}{l}5 \mathrm{~g}\left(17.6 \mathrm{mmol}^{2} \text { of TTIP, } 0.17 \mathrm{~g} \mathrm{(1.5}\right. \\
\text { mmol) } \mathrm{CaCl}_{2}, 316 \mu \mathrm{L}(17.6 \mathrm{mmol}) \\
\text { water }\end{array}$ & $\begin{array}{l}\text { Colorless } \\
\text { block crystals }\end{array}$ & $28 \%$ & $\begin{array}{l}\text { Anal. Calcd for } \mathrm{C}_{54} \mathrm{H}_{127} \mathrm{O}_{32} \mathrm{ClCaTi}_{11} \text { : } \\
\mathrm{Ti}, 27.86 \% ; \mathrm{Ca}, 2.121 \% \text {. Found: } \\
29.61 \% \text {; Ca, 2.329\%. }\end{array}$ & $\begin{array}{l}\text { SXRD, PXRD, } \\
\text { FTIR }\end{array}$ \\
\hline 13 & $\begin{array}{l}\mathrm{Ti}_{11} \mathrm{O}_{14} \mathrm{CaBr}\left(\mathrm{O}^{i}\right. \\
\mathrm{Pr})_{17}\left(\mathrm{HO}^{i} \mathrm{Pr}\right)_{2}\end{array}$ & $\begin{array}{l}5 \mathrm{~g}(17.6 \mathrm{mmol}) \text { of } \mathrm{TTIP}, 0.3 \mathrm{~g}(1.5 \\
\mathrm{mmol}) \mathrm{CaBr}_{2}, 316 \mu \mathrm{L}(17.6 \mathrm{mmol}) \\
\text { water }\end{array}$ & $\begin{array}{l}\text { Colorless } \\
\text { block crystals }\end{array}$ & $32 \%$ & $\begin{array}{lll}\text { Anal. } & \text { Calcd } & \text { for } \\
\mathrm{C}_{57} \mathrm{H}_{135} \mathrm{O}_{33} \mathrm{BrCaTi}_{11}: \mathrm{Ti}, 26.4 \% & \mathrm{Ca} \text {, } \\
2.01 \% . & \text { Found: } \quad 27.31 \% ; & \mathrm{Ca}, \\
2.259 \% . & & \end{array}$ & $\begin{array}{l}\text { SXRD, PXRD, }{ }^{13} \mathrm{C} \\
\text { NMR, FTIR }\end{array}$ \\
\hline 14 & $\begin{array}{l}\mathrm{Ti}_{11} \mathrm{O}_{14} \mathrm{ZnCl}\left(\mathrm{O}^{i}\right. \\
\mathrm{Pr})_{17}\end{array}$ & $\begin{array}{l}5 \mathrm{~g}(17.6 \mathrm{mmol}) \text { of } \mathrm{TTIP}, 0.2 \mathrm{~g}(1.5 \\
\mathrm{mmol}) \mathrm{ZnCl}_{2}, 316 \mu \mathrm{L}(17.6 \mathrm{mmol}) \\
\text { water }\end{array}$ & $\begin{array}{l}\text { Colorless } \\
\text { block crystals }\end{array}$ & $42 \%$ & $\begin{array}{l}\text { Anal. Calcd for } \mathrm{C}_{51} \mathrm{H}_{119} \mathrm{O}_{31} \mathrm{ClZnTi}_{11} \text { : } \\
\mathrm{Ti}, 28.38 \% ; \mathrm{Zn}, 3.526 \% \text {. Found: Ti, } \\
\text { 31.47\%; } \mathrm{Zn}, 3.374 \% \text {. }\end{array}$ & $\begin{array}{l}\text { SXRD, PXRD, }{ }^{13} \mathrm{C} \\
\text { NMR, FTIR }\end{array}$ \\
\hline 15 & $\begin{array}{l}\mathrm{Ti}_{11} \mathrm{O}_{14} \mathrm{ZnBr}\left(\mathrm{O}^{i}\right. \\
\mathrm{Pr})_{17}\end{array}$ & $\begin{array}{l}5 \mathrm{~g}(17.6 \mathrm{mmol}) \text { of } \mathrm{TTIP}, 0.34 \mathrm{~g}(1.5 \\
\mathrm{mmol}) \mathrm{ZnBr}_{2}, 316 \mu \mathrm{L}(17.6 \mathrm{mmol}) \\
\text { water }\end{array}$ & $\begin{array}{l}\text { Colorless } \\
\text { block crystals }\end{array}$ & $38 \%$ & $\begin{array}{l}\text { Anal. Calcd } r \text { for } \\
\mathrm{C}_{51} \mathrm{H}_{119} \mathrm{O}_{31} \mathrm{BrZnTi}_{11}: \mathrm{Ti}, \quad 27.72 \% \\
\mathrm{Zn}, 3.443 \% \text {. Found: } \mathrm{Ti}, 29.34 \% ; \mathrm{Zn} \text {, } \\
\text { 3.189\%. }\end{array}$ & $\begin{array}{l}\text { SXRD, PXRD, }{ }^{13} \mathrm{C} \\
\text { NMR, FTIR }\end{array}$ \\
\hline 16 & $\begin{array}{l}\mathrm{Ti}_{11} \mathrm{O}_{14} \mathrm{CdCl}\left(\mathrm{O}^{i}\right. \\
\mathrm{Pr})_{17}\end{array}$ & $\begin{array}{l}5 \mathrm{~g}(17.6 \mathrm{mmol}) \text { of TTIP, } 0.32 \mathrm{~g}(1.5 \\
\mathrm{mmol}) \mathrm{CdCl}_{2} \cdot 1.5 \mathrm{H}_{2} \mathrm{O}, 276 \mu \mathrm{L}(15.4 \\
\text { mmol) water }\end{array}$ & $\begin{array}{l}\text { Colorless } \\
\text { block crystals }\end{array}$ & $24 \%$ & 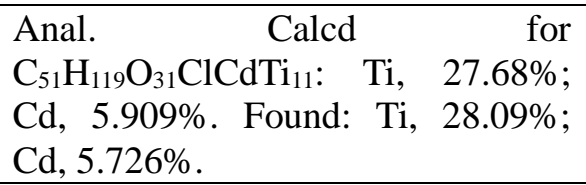 & $\begin{array}{l}\text { SXRD, } \\
\text { FTIR }\end{array}$ \\
\hline 17 & $\begin{array}{l}\mathrm{Ti}_{11} \mathrm{O}_{14} \mathrm{CdBr}\left(\mathrm{O}^{i}\right. \\
\mathrm{Pr})_{17}\end{array}$ & $\begin{array}{l}5 \mathrm{~g}(17.6 \mathrm{mmol}) \text { of } \mathrm{TTIP}, 0.41 \mathrm{~g}(1.5 \\
\mathrm{mmol}) \mathrm{CdBr}_{2}, 316 \mu \mathrm{L}(17.6 \mathrm{mmol}) \\
\text { water }\end{array}$ & $\begin{array}{l}\text { Colorless } \\
\text { block crystals }\end{array}$ & $18 \%$ & 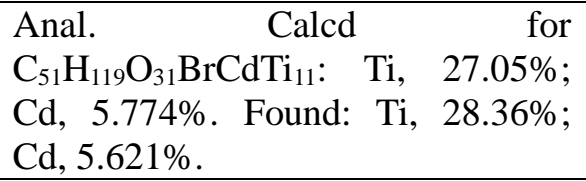 & $\begin{array}{l}\text { SXRD, } \\
\text { FTIR }\end{array}$ \\
\hline
\end{tabular}

\section{Single crystal X-ray crystallographic data}

Table S2a. Single crystal X-ray crystallographic data 


\begin{tabular}{|c|c|c|c|c|c|c|}
\hline & $\left\{\mathbf{T i}_{12}\right\}$ & $\left\{\mathbf{T i}_{11}\right\}$ & $\left\{\mathrm{Ti}_{11} \mathrm{CoCl}\right\}$ & $\left\{\mathrm{Ti}_{11} \mathrm{CoBr}\right\}$ & $\left\{\mathrm{Ti}_{11} \mathrm{CoI}\right\}$ & $\left\{\mathrm{Ti}_{11} \mathrm{Co}\left(\mathrm{NO}_{3}\right)\right\}$ \\
\hline Formula & $\mathrm{C}_{48} \mathrm{H}_{112} \mathrm{O}_{32} \mathrm{Ti}_{12}$ & $\mathrm{C}_{54} \mathrm{H}_{126} \mathrm{O}_{32} \mathrm{Ti}_{11}$ & $\mathrm{C}_{51} \mathrm{H}_{119} \mathrm{ClCoO}_{31} \mathrm{Ti}_{11}$ & $\mathrm{C}_{51} \mathrm{H}_{119} \mathrm{BrCoO}_{31} \mathrm{Ti}_{11}$ & $\mathrm{C}_{51} \mathrm{H}_{119} \mathrm{ICoO}_{31} \mathrm{Ti}_{11}$ & $\mathrm{C}_{102} \mathrm{H}_{238} \mathrm{~N}_{2} \mathrm{Co}_{2} \mathrm{O}_{68} \mathrm{Ti}_{22}$ \\
\hline $\begin{array}{l}\text { Formula } \\
\text { weight }\end{array}$ & 1776.17 & 1814.44 & 1849.73 & 1894.19 & 1941.18 & 3752.59 \\
\hline Crystal system & monoclinic & monoclinic & monoclinic & monoclinic & monoclinic & monoclinic \\
\hline Space group & $\mathrm{P} 2_{1} / \mathrm{c}$ & $\mathrm{P} 2_{1} / \mathrm{n}$ & $\mathrm{Pn}$ & $\mathrm{P} \mathrm{n}$ & $\mathrm{Pn}$ & $\mathrm{Pc}$ \\
\hline Temperature/K & 293 & 173.15 & 173.15 & 173.15 & 173.15 & 173.15 \\
\hline $\mathrm{a}(\AA)$ & $13.049(4)$ & $16.302(3)$ & $15.047(3)$ & $15.0664(16)$ & $15.0662(4)$ & $26.935(17)$ \\
\hline b $(\AA)$ & $20.954(7)$ & $14.391(3)$ & $13.919(3)$ & $13.9101(14)$ & $13.9583(4)$ & $13.625(7)$ \\
\hline$c(\AA)$ & $16.953(4)$ & $19.729(5)$ & $20.904(5)$ & $20.852(2)$ & $20.9181(5)$ & $26.060(10)$ \\
\hline$\alpha\left(^{\circ}\right)$ & 90 & 90 & 90 & 90 & 90 & 90 \\
\hline$\beta\left(^{\circ}\right)$ & $121.536(17)$ & $91.356(4)$ & $90.123(2)$ & 90 & 90 & $112.47(4)$ \\
\hline$\gamma\left({ }^{\circ}\right)$ & 90 & 90 & 90 & 90 & 90 & 90 \\
\hline $\mathrm{V}(\AA 3)$ & $3951(2)$ & $4627.1(14)$ & $4378.0(17)$ & $4370.1(8)$ & $4399.0(2)$ & $8838(8)$ \\
\hline $\mathrm{Z}$ & 2 & 2 & 2 & 2 & 2 & 2 \\
\hline Dcalc $(\mathrm{g} / \mathrm{cm} 3)$ & 1.493 & 1.302 & 1.449 & 1.44 & 1.466 & 1.41 \\
\hline $\begin{array}{l}\text { Abs. Coeff. T } \\
\left(\mathrm{mm}^{-1}\right)\end{array}$ & 1.214 & 0.960 & 1.228 & 1.654 & 1.54 & 10.023 \\
\hline $\begin{array}{l}\text { Total } \\
\text { reflections }\end{array}$ & 21730 & 22704 & 25516 & 18979 & 20111 & 47871 \\
\hline $\operatorname{Min}-\max \theta\left(^{\circ}\right)$ & $\begin{array}{l}3.424 \text { to } \\
49.998 \\
\end{array}$ & 3.776 to 50 & 5.414 to 54.96 & 2.928 to 55.134 & 5.568 to 54.992 & 3.55 to 127.512 \\
\hline $\begin{array}{l}\text { Unique } \\
\text { reflections }\end{array}$ & 18708 & 22624 & 24956 & 18414 & 19606 & 46685 \\
\hline $\begin{array}{l}\text { Calculated } \\
\text { reflection }(I> \\
4 \sigma)\end{array}$ & 3868 & 3083 & 10663 & 6153 & 6605 & 11488 \\
\hline $\mathrm{R} 1[\mathrm{I}>=2 \sigma] \mathrm{c}$ & 0.0897 & 0.0966 & 0.0579 & 0.061 & 0.0635 & 0.0947 \\
\hline wR2(all data)d & 0.2920 & 0.2842 & 0.1469 & 0.1624 & 0.1486 & 0.2894 \\
\hline Rint & 0.1530 & 0.1493 & 0.0454 & 0.0505 & 0.0611 & 0.1085 \\
\hline $\begin{array}{l}\text { Goodness of } \\
\text { fit on F2 }\end{array}$ & 1.004 & 0.929 & 1.017 & 0.881 & 0.841 & 1.043 \\
\hline Parameters & 431 & 416 & 908 & 741 & 736 & 1000 \\
\hline Restraints & 67 & 97 & 93 & 74 & 26 & 25 \\
\hline Largest diff. & $1.26 /-1.70$ & $0.91 /-0.79$ & $1.16 /-0.70$ & $0.81 /-0.51$ & $0.92 /-0.50$ & $1.56 /-0.82$ \\
\hline
\end{tabular}


Table S2b. Single crystal X-ray crystallographic data

\begin{tabular}{|c|c|c|c|c|c|c|}
\hline & $\left\{\mathrm{Ti}_{11} \mathrm{NiCl}\right\}$ & $\left\{\mathrm{Ti}_{11} \mathrm{NiBr}\right\}$ & $\left\{\mathrm{Ti}_{11} \mathrm{NiI}\right\}$ & $\left\{\mathrm{Ti}_{11} \mathrm{MgCl}\right\}$ & $\left\{\mathrm{Ti}_{11} \mathrm{MgBr}\right\}$ & $\left\{\mathrm{Ti}_{11} \mathrm{CaCl}\right\}$ \\
\hline Formula & $\mathrm{C}_{54} \mathrm{H}_{127} \mathrm{ClNiO}_{32} \mathrm{Ti}_{11}$ & $\mathrm{C}_{51} \mathrm{H}_{119} \mathrm{BrNiO}_{31} \mathrm{Ti}_{11}$ & $\mathrm{C}_{51} \mathrm{H}_{119} \mathrm{INiO}_{31} \mathrm{Ti}_{11}$ & $\mathrm{C}_{51} \mathrm{H}_{119} \mathrm{ClMgO}_{31} \mathrm{Ti}_{11}$ & $\mathrm{C}_{51} \mathrm{H}_{119} \mathrm{BrMgO}_{31} \mathrm{Ti}_{11}$ & $\mathrm{C}_{57} \mathrm{H}_{135} \mathrm{ClCaO}_{33} \mathrm{Ti}_{11}$ \\
\hline Formula weight & 1909.61 & 1893.97 & 1940.96 & 1815.11 & 1859.57 & 1951.07 \\
\hline Crystal system & monoclinic & monoclinic & monoclinic & monoclinic & monoclinic & monoclinic \\
\hline Space group & $\mathrm{Pn}$ & $\mathrm{Pn}$ & $\mathrm{Pn}$ & $\mathrm{Pc}$ & $\mathrm{Pn}$ & $\mathrm{P} 22_{1} / \mathrm{c}$ \\
\hline Temperature/K & 173.15 & 173.15 & 173.15 & 173.15 & 173.15 & 173.15 \\
\hline $\mathrm{a}(\AA)$ & $15.0469(9)$ & $15.118(3)$ & $15.1113(5)$ & $15.050(8)$ & $15.110(3)$ & $15.036(4)$ \\
\hline $\mathrm{b}(\AA)$ & $13.9297(9)$ & $13.918(3)$ & $13.9459(4)$ & $13.864(8)$ & $14.173(3)$ & $15.163(4)$ \\
\hline $\mathrm{c}(\AA)$ & $20.9419(14)$ & $20.887(5)$ & $20.8851(6)$ & $25.664(11)$ & $20.714(5)$ & $39.981(10)$ \\
\hline$\alpha\left(^{\circ}\right)$ & 90 & 90 & 90 & 90 & 90 & 90 \\
\hline$\beta\left(^{\circ}\right)$ & 90 & $90.054(2)$ & $90.141(2)$ & $125.86(2)$ & $90.234(2)$ & 92.091(3) \\
\hline$\gamma\left({ }^{\circ}\right)$ & 90 & 90 & 90 & 90 & 90 & 90 \\
\hline $\mathrm{V}(\AA 3)$ & $4389.4(5)$ & 4395.1(17) & $4401.3(2)$ & $4340(4)$ & $4435.8(17)$ & $9109(4)$ \\
\hline $\mathrm{Z}$ & 2 & 2 & 2 & 2 & 2 & 4 \\
\hline Dcalc $\left(\mathrm{g} / \mathrm{cm}^{3}\right)$ & 1.445 & 1.431 & 1.465 & 1.389 & 1.392 & 1.423 \\
\hline $\begin{array}{l}\text { Abs. Coeff. T } \\
\left(\mathrm{mm}^{-1}\right)\end{array}$ & 1.251 & 1.67 & 1.564 & 1.059 & 1.456 & 1.064 \\
\hline Total reflections & 25461 & 27688 & 32955 & 36596 & 28282 & 60437 \\
\hline Min-max $\theta\left(^{\circ}\right)$ & 5.414 to 55.318 & 4.876 to 55.046 & 5.392 to 55.046 & 3.53 to 54.896 & 4.872 to 55.492 & 4.952 to 54.972 \\
\hline $\begin{array}{l}\text { Unique } \\
\text { reflections }\end{array}$ & 24686 & 27134 & 32074 & 35654 & 27632 & 59008 \\
\hline $\begin{array}{l}\text { Calculated } \\
\text { reflection }(I>4 \sigma)\end{array}$ & 5935 & 9066 & 5603 & 9841 & 6503 & 8526 \\
\hline $\mathrm{R} 1[\mathrm{I}>=2 \sigma] \mathrm{c}$ & 0.0604 & 0.0736 & 0.062 & 0.0692 & 0.1035 & 0.0823 \\
\hline wR2 (all data)d & 0.1235 & 0.2012 & 0.1452 & 0.2238 & 0.3603 & 0.2747 \\
\hline Rint & 0.0802 & 0.074 & 0.1231 & 0.0707 & 0.0593 & 0.0847 \\
\hline
\end{tabular}




\begin{tabular}{|c|c|c|c|c|c|c|}
\hline $\begin{array}{l}\text { Goodness of fit } \\
\text { on } \mathrm{F}^{2}\end{array}$ & 0.772 & 0.976 & 0.829 & 0.996 & 1.024 & 1.03 \\
\hline Parameters & 646 & 888 & 637 & 867 & 686 & 966 \\
\hline Restraints & 43 & 116 & 44 & 58 & 46 & 141 \\
\hline $\begin{array}{l}\text { Largest diff. } \\
\text { peak/hole }\left(\mathrm{e} \AA^{3}\right)\end{array}$ & $0.49 /-0.44$ & $1.11 /-0.60$ & $0.77 /-0.56$ & $1.42 /-0.55$ & $2.85 /-1.44$ & $1.04 /-0.59$ \\
\hline
\end{tabular}

Table S2c. Single crystal X-ray crystallographic data

\begin{tabular}{|c|c|c|c|c|c|}
\hline & $\left\{\mathrm{Ti}_{11} \mathrm{CaBr}\right\}$ & $\left\{\mathrm{Ti}_{11} \mathrm{ZnCl}\right\}$ & $\left\{\mathrm{Ti}_{11} \mathrm{ZnBr}\right\}$ & $\left\{\mathrm{Ti}_{11} \mathrm{CdCl}\right\}$ & $\left\{\mathrm{Ti}_{11} \mathrm{CdBr}\right\}$ \\
\hline Formula & $\mathrm{C}_{60} \mathrm{H}_{141} \mathrm{BrCaO}_{34} \mathrm{Ti}_{11}$ & $\mathrm{C}_{54} \mathrm{H}_{127} \mathrm{ClZnO}_{32} \mathrm{Ti}_{11}$ & $\mathrm{C}_{51} \mathrm{H}_{119} \mathrm{BrZnO}_{31} \mathrm{Ti}_{11}$ & $\mathrm{C}_{51} \mathrm{H}_{119} \mathrm{ClCdO}_{31} \mathrm{Ti}_{11}$ & $\mathrm{C}_{51} \mathrm{H}_{119} \mathrm{BrCdO}_{31} \mathrm{Ti}_{11}$ \\
\hline Formula weight & 2053.61 & 1916.27 & 1900.63 & 1903.2 & 1947.66 \\
\hline Crystal system & orthorhombic & monoclinic & monoclinic & monoclinic & monoclinic \\
\hline Space group & $\mathrm{P}$ ca2 $2_{1}$ & $\mathrm{Pn}$ & $\mathrm{Pn}$ & $\mathrm{Pn}$ & $\mathrm{Pn}$ \\
\hline Temperature/K & 173.15 & 173.15 & 173.15 & 173.15 & 173.15 \\
\hline $\mathrm{a}(\AA)$ & $26.4617(9)$ & $15.044(4)$ & $15.0608(4)$ & $20.979(9)$ & $15.081(4)$ \\
\hline $\mathrm{b}(\AA)$ & $14.9124(6)$ & $13.924(4)$ & $13.9572(5)$ & $13.904(6)$ & $13.925(3)$ \\
\hline$c(\AA)$ & $24.3861(10)$ & $20.850(5)$ & $20.8997(6)$ & $15.104(6)$ & $20.959(5)$ \\
\hline$\alpha\left(^{\circ}\right)$ & 90 & 90 & 90 & 90 & 90 \\
\hline$\beta\left(^{\circ}\right)$ & 90 & $90.041(2)$ & 90 & 90 & 90 \\
\hline$\gamma\left({ }^{\circ}\right)$ & 90 & 90 & 90 & 90 & 90 \\
\hline $\mathrm{V}\left(\AA^{3}\right)$ & $9622.9(6)$ & $4367(2)$ & $4393.3(2)$ & $4406(3)$ & 4401.3(18) \\
\hline $\mathrm{Z}$ & 4 & 2 & 2 & 2 & 2 \\
\hline Dcalc $\left(\mathrm{g} / \mathrm{cm}^{3}\right)$ & 1.417 & 1.457 & 1.437 & 1.435 & 1.47 \\
\hline Abs. Coeff. T $\left(\mathrm{mm}^{-1}\right)$ & 1.398 & 1.316 & 1.729 & 1.27 & 1.695 \\
\hline Total reflections & 67663 & 25247 & 22655 & 25524 & 23616 \\
\hline Min-max $\theta\left(^{\circ}\right)$ & 5.302 to 55.066 & 5.582 to 54.89 & 5.838 to 55.046 & 5.394 to 54.94 & 5.402 to 55.074 \\
\hline Unique reflections & 65438 & 24725 & 21995 & 24619 & 22869 \\
\hline $\begin{array}{l}\text { Calculated reflection } \\
(\mathrm{I}>4 \sigma)\end{array}$ & 9095 & 10051 & 6932 & 9358 & 7763 \\
\hline $\mathrm{R} 1[\mathrm{I}>=2 \sigma] \mathrm{c}$ & 0.0681 & 0.0561 & 0.0534 & 0.0472 & 0.0556 \\
\hline
\end{tabular}




\begin{tabular}{|c|c|c|c|c|c|}
\hline wR2(all data)d & 0.1746 & 0.1479 & 0.1243 & 0.1203 & 0.1326 \\
\hline Rint & 0.0962 & 0.0474 & 0.0543 & 0.0374 & 0.0455 \\
\hline Goodness of fit on $\mathrm{F}^{2}$ & 1.058 & 1.033 & 0.824 & 0.892 & 0.888 \\
\hline Parameters & 976 & 903 & 835 & 868 & 827 \\
\hline Restraints & 48 & 75 & 123 & 21 & 17 \\
\hline $\begin{array}{l}\text { Largest diff. peak/hole } \\
\left(\mathrm{e} \AA^{3}\right)\end{array}$ & $0.57 /-0.58$ & $1.06 /-0.73$ & $0.60 /-0.39$ & $0.73 /-0.45$ & $1.41 /-0.46$ \\
\hline
\end{tabular}




\section{UV-vis titration of $\left\{\mathbf{T i}_{11}\right\}$}

For a monolacunary Keggin polyoxometalate, using X-ray diffraction to determine the structure of the lacunary site is difficult because of disorder. Introducing a heteroatom at the defect site can partially solve the problem. For example, to determine the structure of $\mathrm{K}_{9} \mathrm{AlW}_{11} \mathrm{O}_{39}$, it was reacted with one equivalent of $\mathrm{VOSO}_{4}$ (and subsequently $\mathrm{Br}_{2}$ ) to give $\mathrm{K}_{6} \mathrm{AlVW}_{11} \mathrm{O}_{40}$ derivative whose structure was resolved by X-ray diffraction. ${ }^{1}$ On the other hand, using different ligands to stabilize the monolacunary core to constrain the disorder of the lacunary site, can also provide the needed structural information. Previously, Klemperer et al. used the X-ray structure of $\mathrm{Ti}_{11} \mathrm{O}_{13}\left(\mathrm{O}^{i} \mathrm{Pr}\right)_{13}(\mathrm{OEt})_{5}$ derivative for $\mathrm{Ti}_{11} \mathrm{O}_{13}\left(\mathrm{O}^{i} \mathrm{Pr}\right)_{18} .^{2}$

Herein, in order to understand the monolacunary feature of $\mathrm{Ti}_{11} \mathrm{O}_{13}\left(\mathrm{O}^{i} \mathrm{Pr}\right)_{18}$, which is synthesized and crystalized by the optimized protocol in this study (i.e., using alkali halide salt to promote the crystallization and enhance the yield), UV-vis titration of $\mathrm{Ti}_{11} \mathrm{O}_{13}\left(\mathrm{O}^{i} \mathrm{Pr}\right)_{18}$ is performed, in addition with the $\mathrm{X}$-ray diffraction and the spectroscopic studies like ${ }^{13} \mathrm{C}$ NMR and FTIR. For this, to a $2.2 \mathrm{mM}\left\{\mathrm{Ti}_{11}\right\}$ in isopropanol, anhydrous $\mathrm{CoCl}_{2}$ is incrementally added, and UV-vis spectra are measured. Reaction of $\mathrm{Ti}_{11} \mathrm{O}_{13}\left(\mathrm{O}^{i} \mathrm{Pr}\right)_{18}$ gives $\mathrm{Ti}_{11} \mathrm{O}_{14} \mathrm{CoCl}\left(\mathrm{O}^{i} \mathrm{Pr}\right)_{17}$ and ${ }^{i} \mathrm{PrCl}$, as indicated by IR and GC-MS (eq S1). Due to the different spectra of $\mathrm{Ti}_{11} \mathrm{O}_{14} \mathrm{CoCl}(\mathrm{O} P r){ }_{17}$ and $\mathrm{CoCl}_{2}$ (Figure S1A), plotting the absorbance of the solution (at $675 \mathrm{~nm}$ ) as a function of added $\mathrm{CoCl}_{2}$ reveals a 1:1 complexation reaction (Figure S1B).

$$
\mathrm{Ti}_{11} \mathrm{O}_{13}\left(\mathrm{O}{ }^{i} \mathrm{Pr}\right)_{18}+\mathrm{CoCl}_{2} \rightarrow \mathrm{Ti}_{11} \mathrm{O}_{14} \mathrm{CoCl}(\mathrm{O} \mathrm{Pr})_{17}+{ }^{i} \mathrm{PrCl}
$$

A

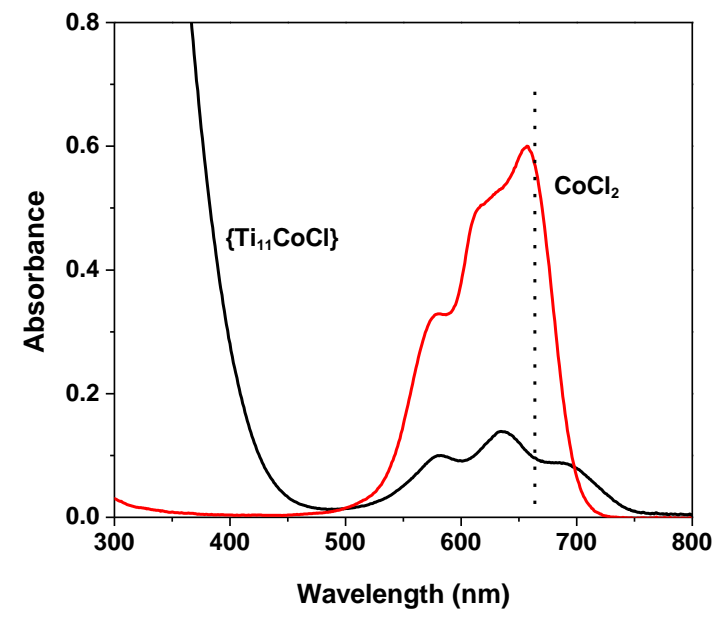

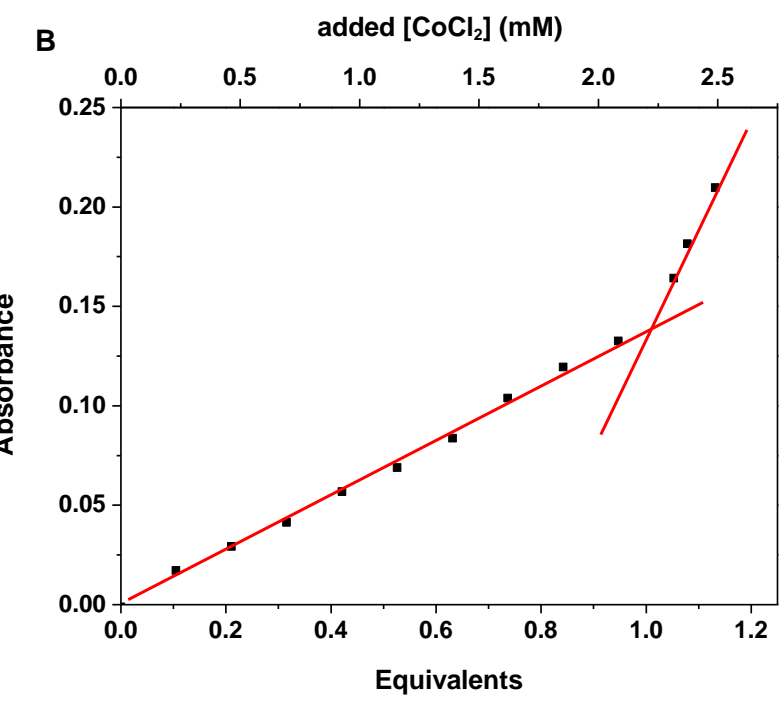

Figure S1. UV-vis titration of $\left\{\mathrm{Ti}_{11}\right\}(2.2 \mathrm{mM})$ with $\mathrm{CoCl}_{2}$ : (A) spectra of $\left\{\mathrm{Ti}_{11} \mathrm{CoCl}\right\}$ and $\mathrm{CoCl}_{2}$ in isopropanol and (B) absorbance (at $675 \mathrm{~nm}$ ) of the mixture as functions of added equivalents of $\mathrm{CoCl}_{2}$.

1. Weinstock, I. A.; Cowan, J. J.; Barbuzzi, E. M. G.; Zeng, H. D.; Hill, C. L., Equilibria between a and b Isomers of Keggin Heteropolytungstates. J. Am. Chem. Soc. 1999, 121, 4608-4617..

2. Day, V. W.; Eberspacher, T. A.; Klemperer, W. G.; Park, C. W., Dodecatitanates: A New Family of Stable Polyoxotitanates. J. Am. Chem. Soc. 1993, 115, (18), 8469-8470.. 
4. Powder X-ray diffraction of $\left\{\mathrm{Ti}_{11} \mathrm{ML}\right\}$.
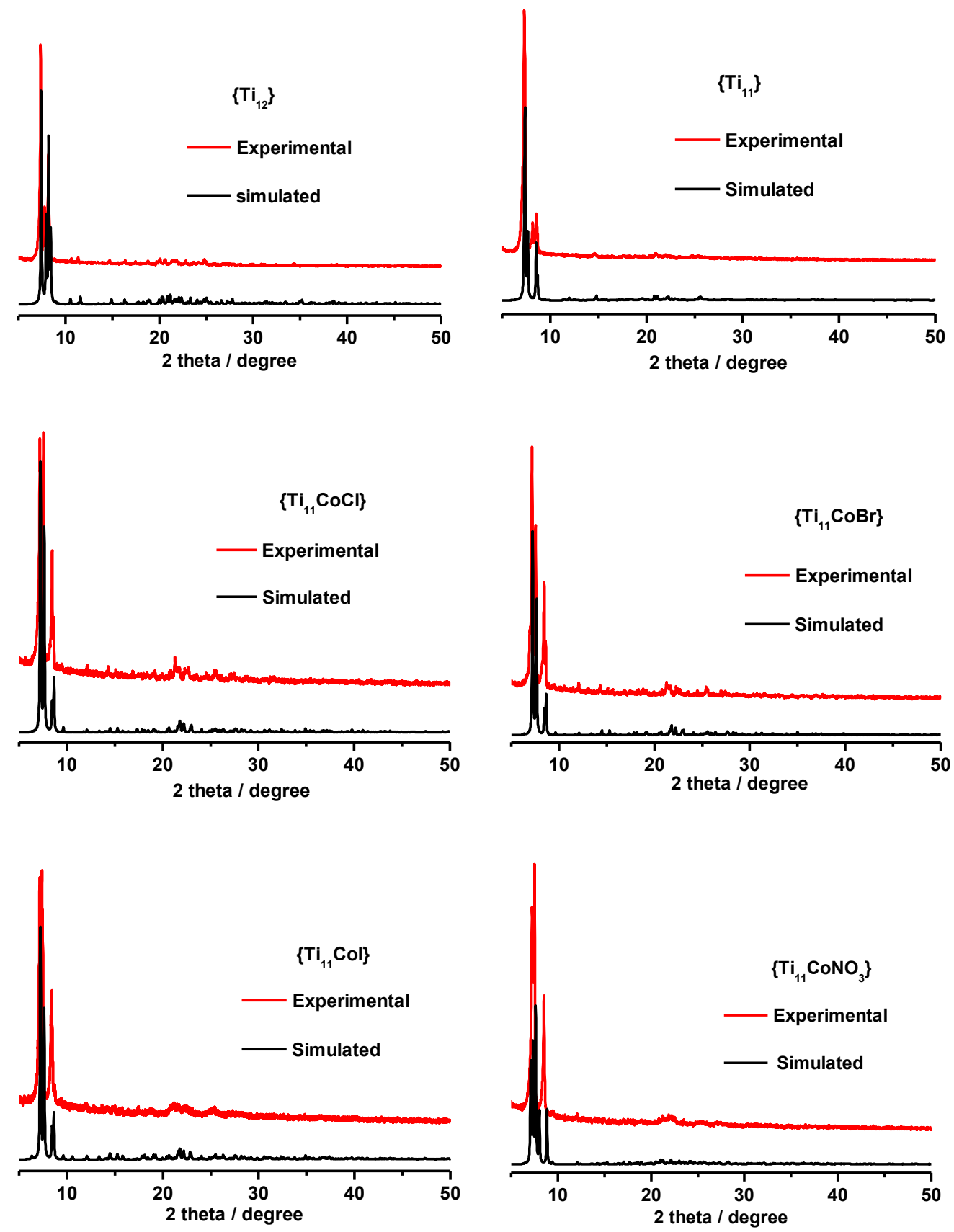

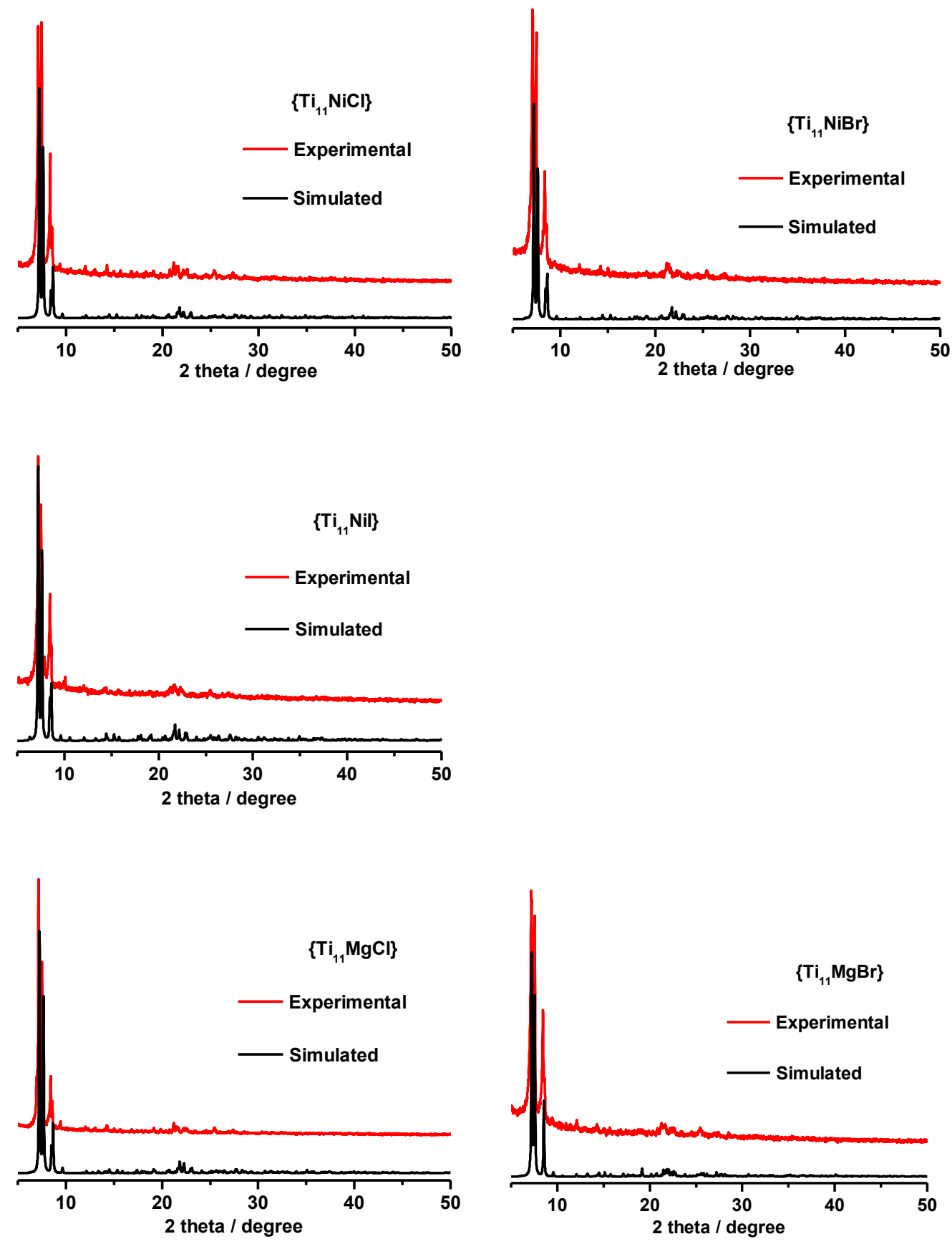

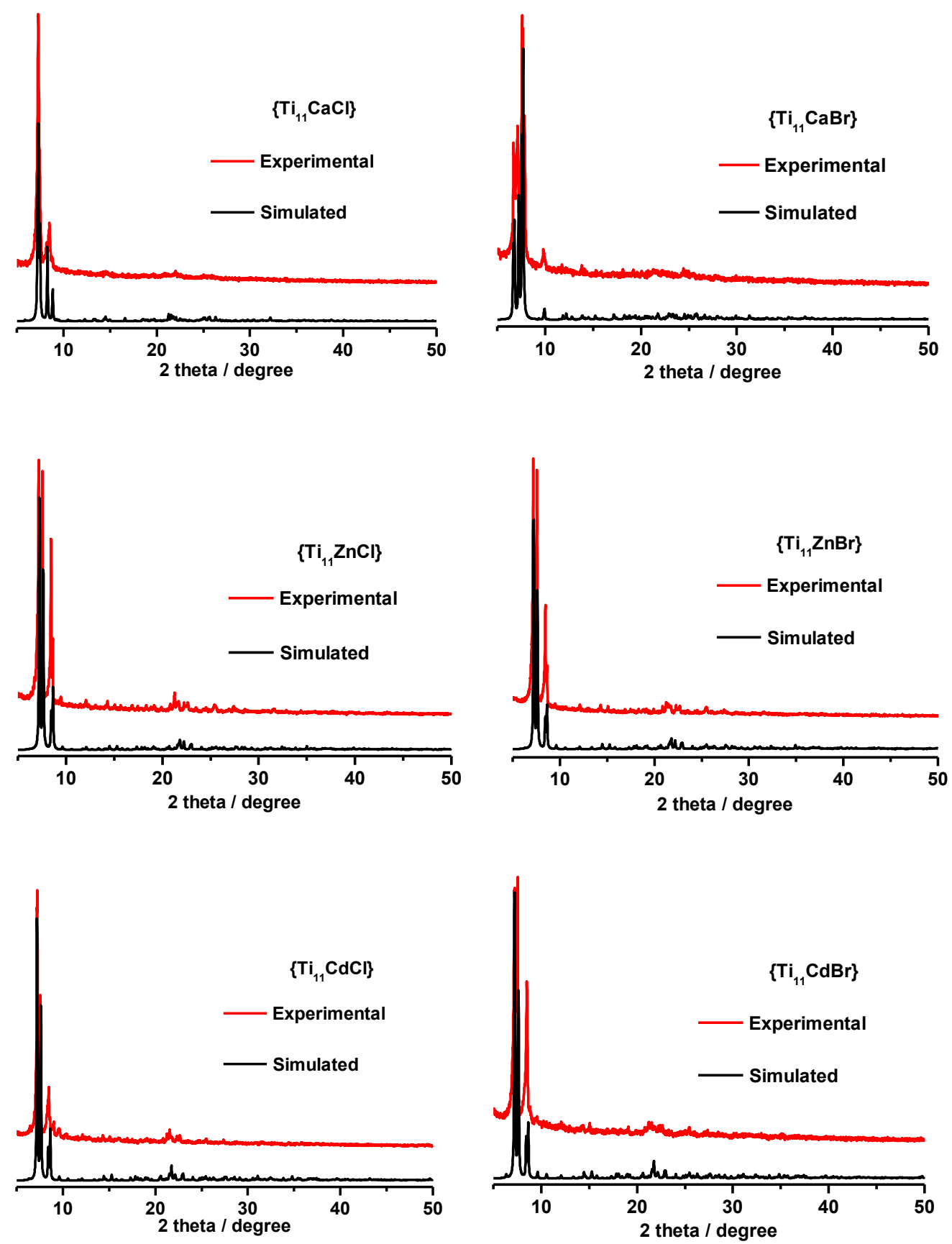

Figure S2. Powder XRD of $\left\{\mathrm{Ti}_{11} \mathrm{ML}\right\}$.

\section{Fourier transform IR spectra}



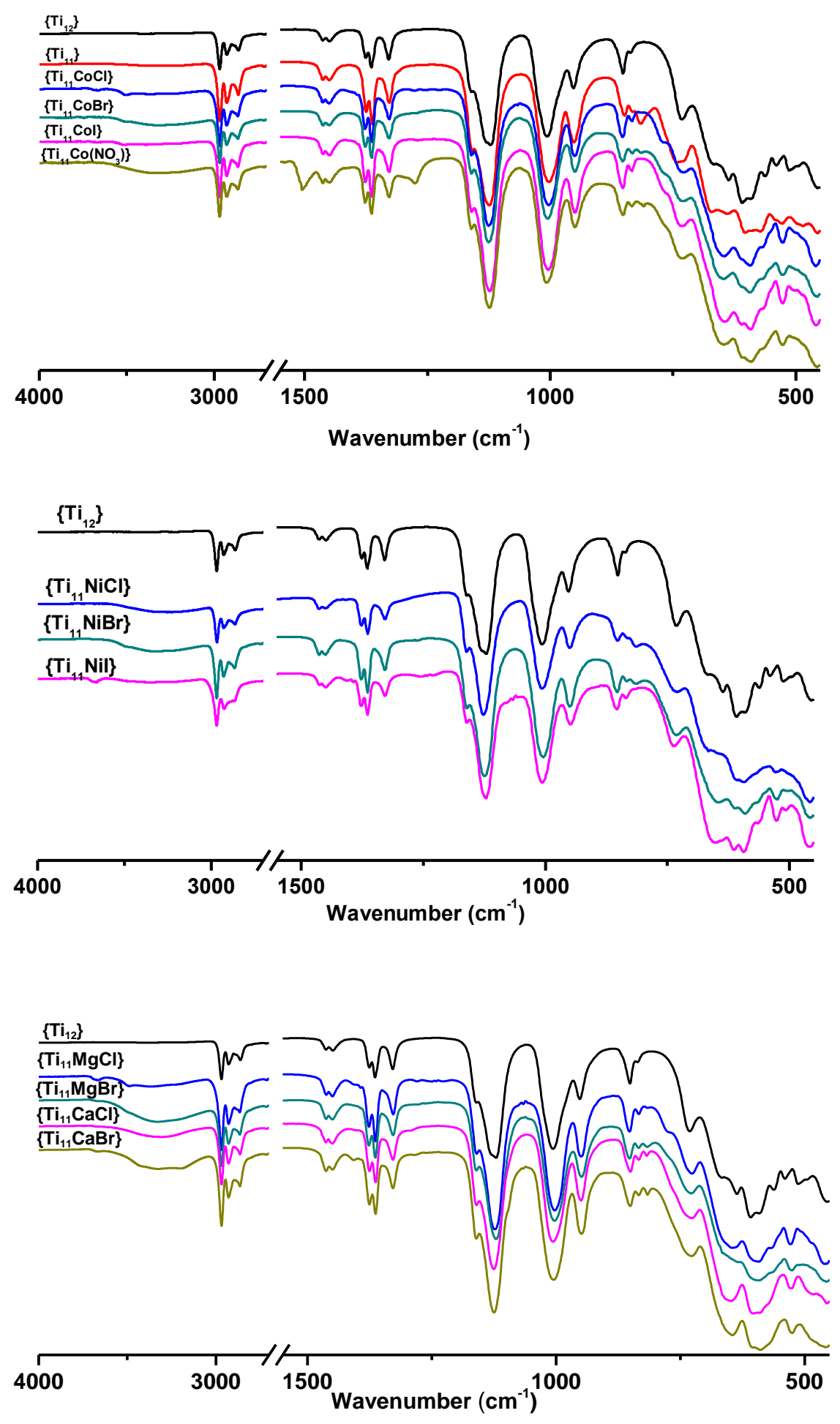


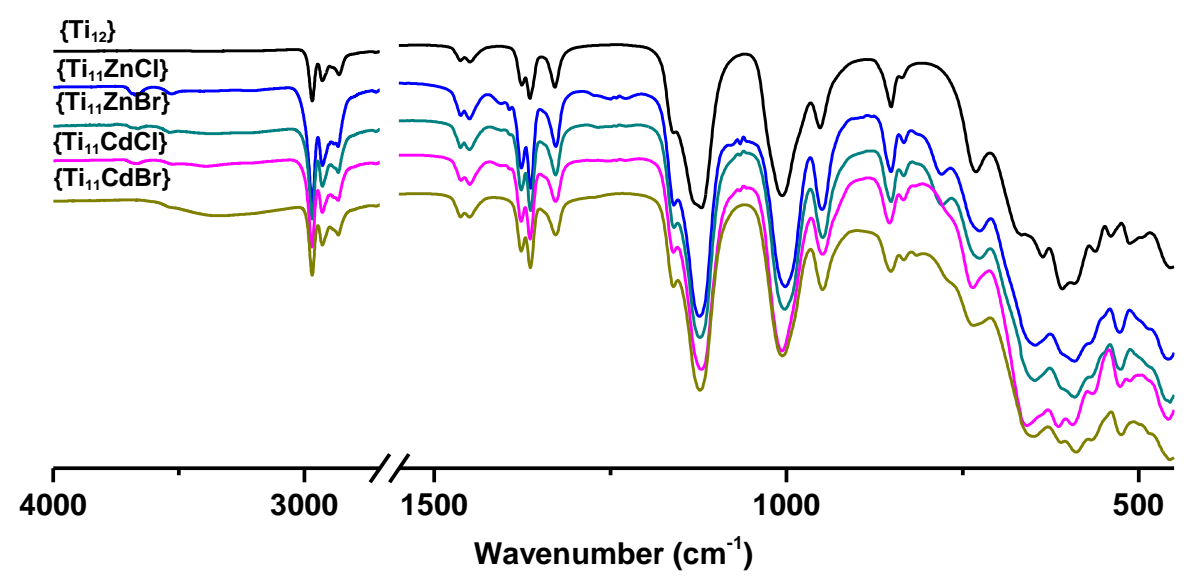

Figure S3. IR spectra of $\left\{\mathrm{Ti}_{12}\right\},\left\{\mathrm{Ti}_{11}\right\}$ and $\left\{\mathrm{Ti}_{11} \mathrm{ML}\right\}$ in solid form.

\section{6. ${ }^{13} \mathrm{C}$ NMR spectra and ${ }^{1} \mathrm{H}$ NMR spectra}

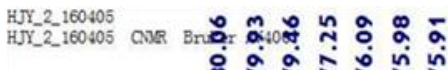

迆

๓)

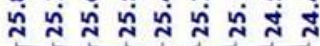

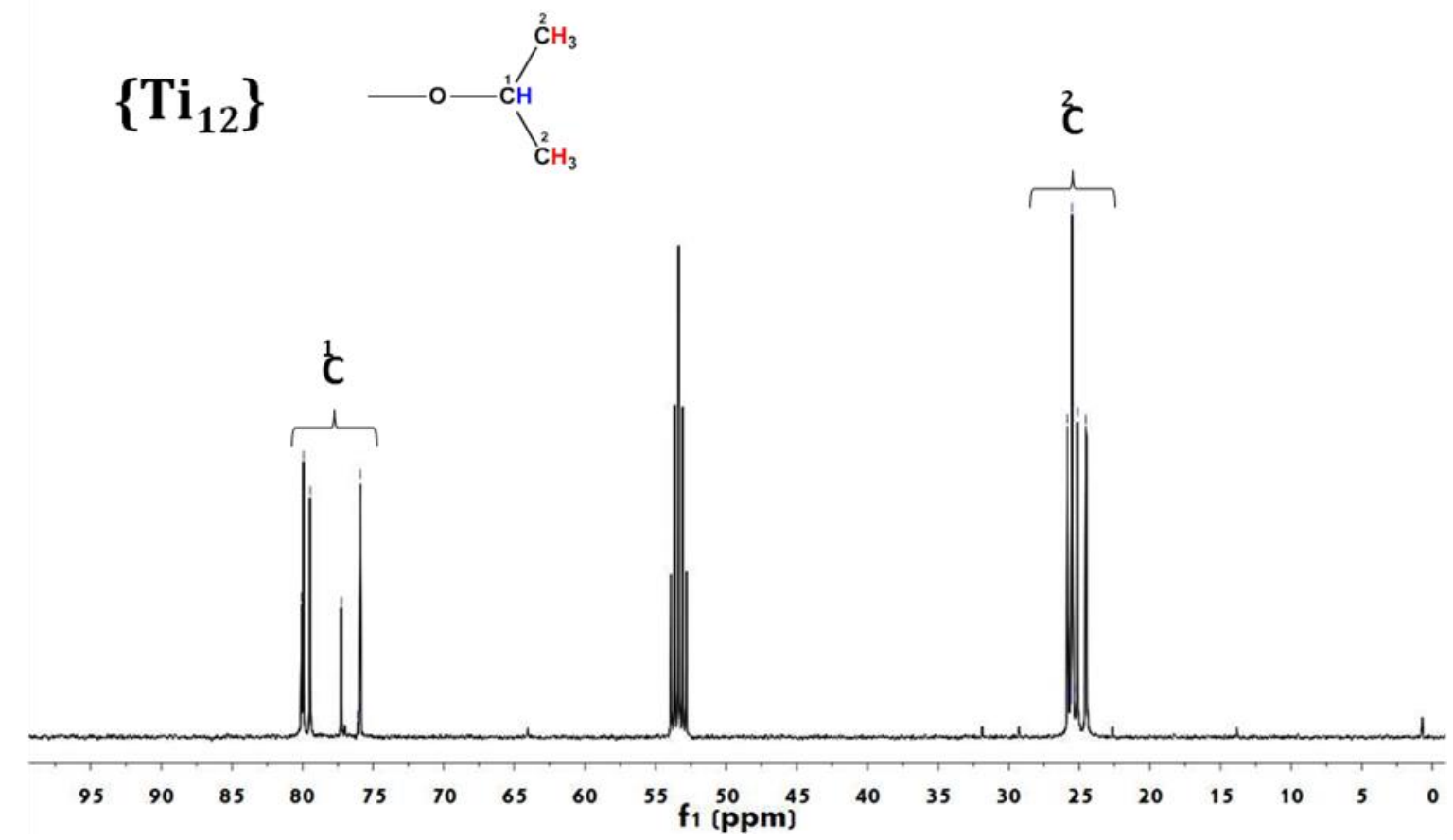



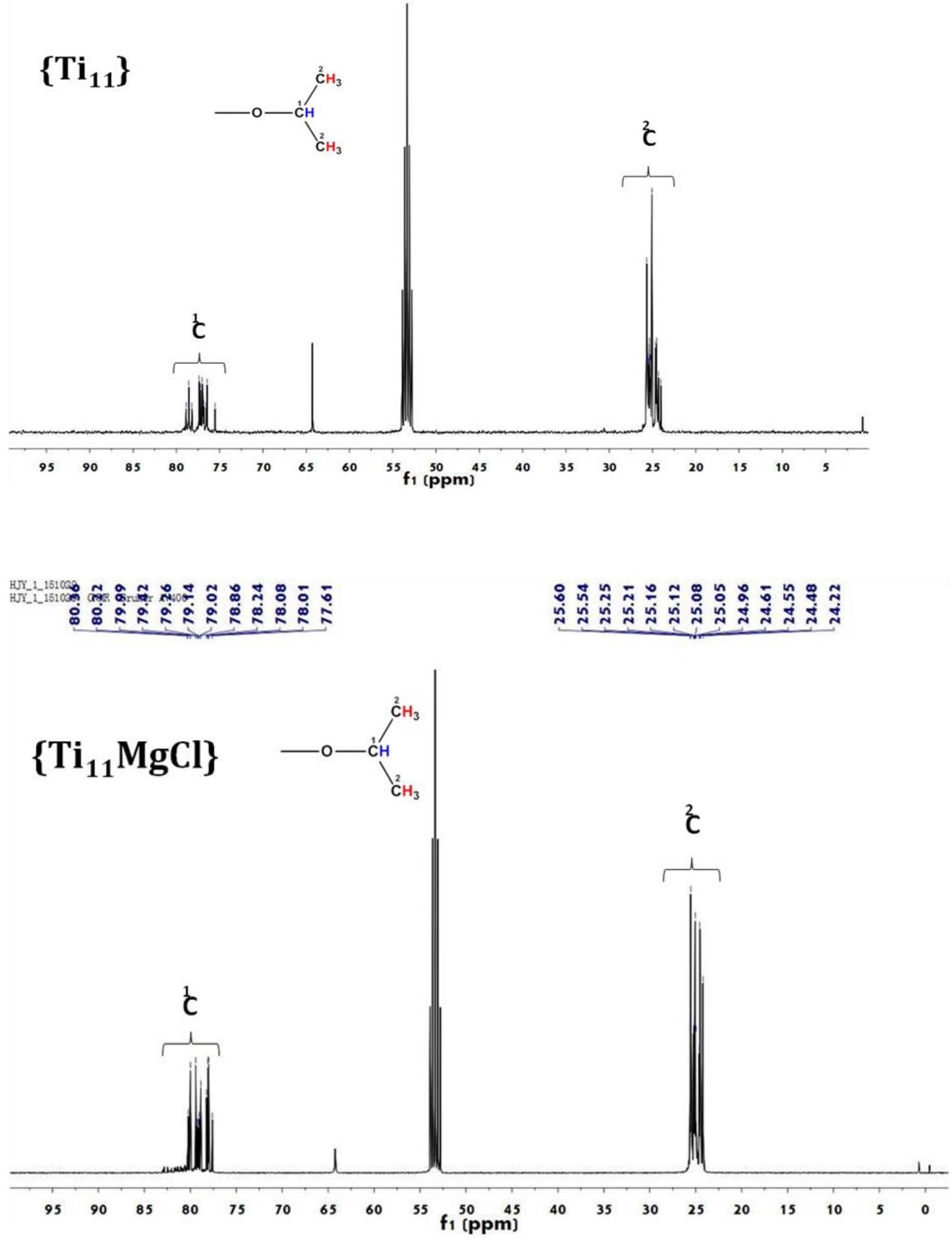
$\left\{\mathrm{Ti}_{11} \mathrm{CaBr}\right\}$

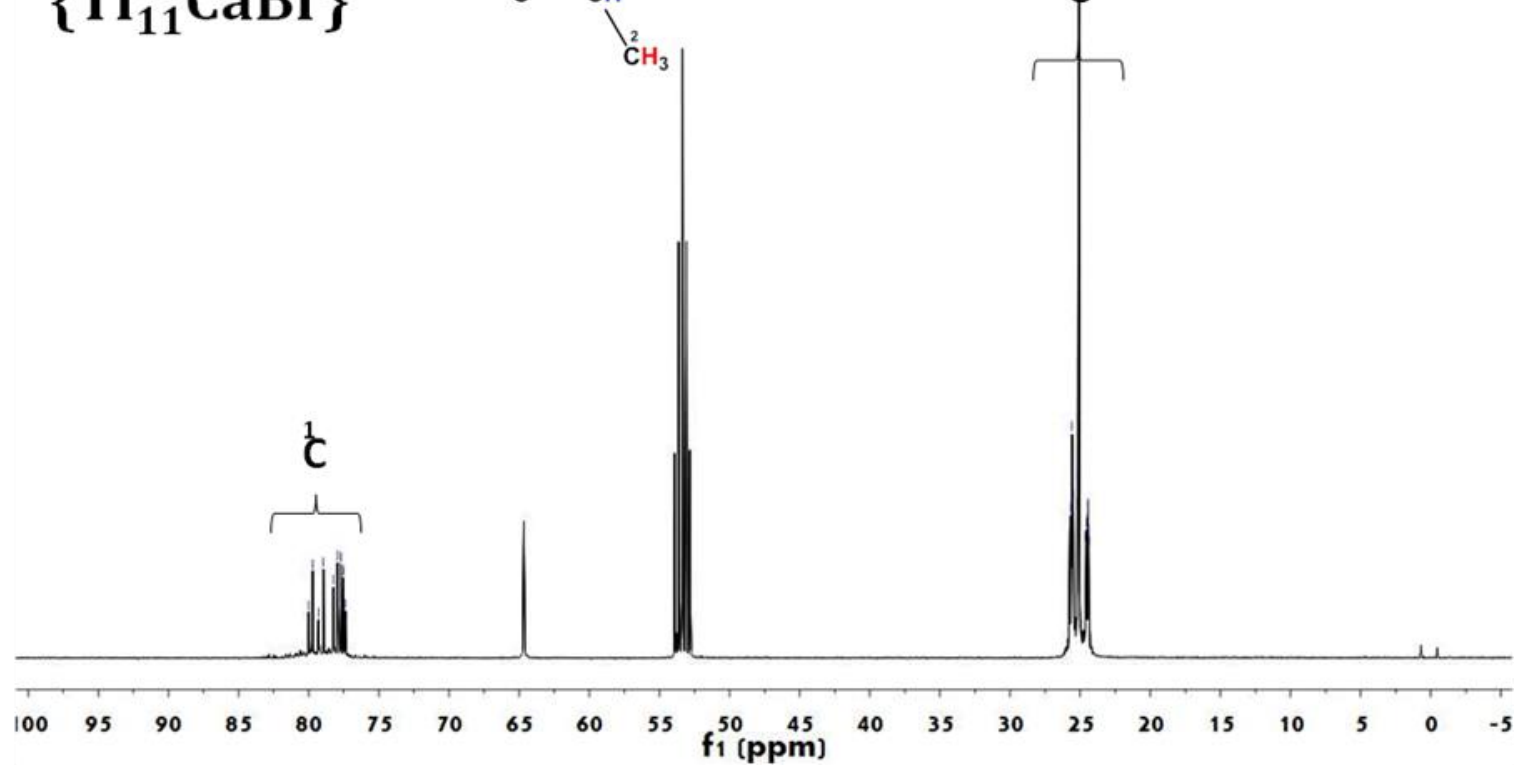

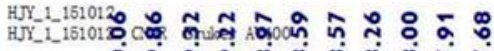

角

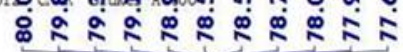

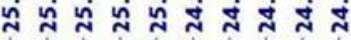

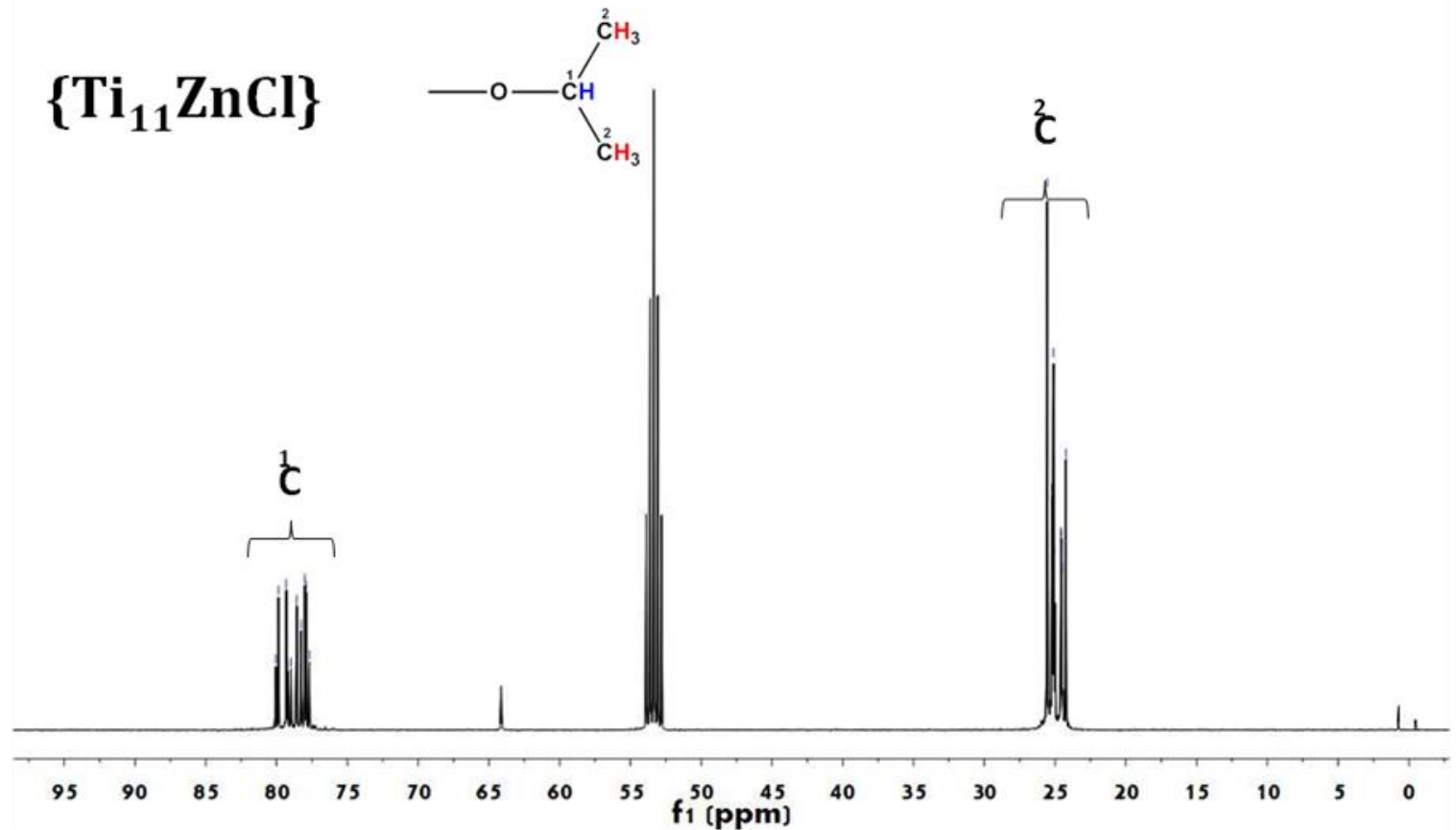




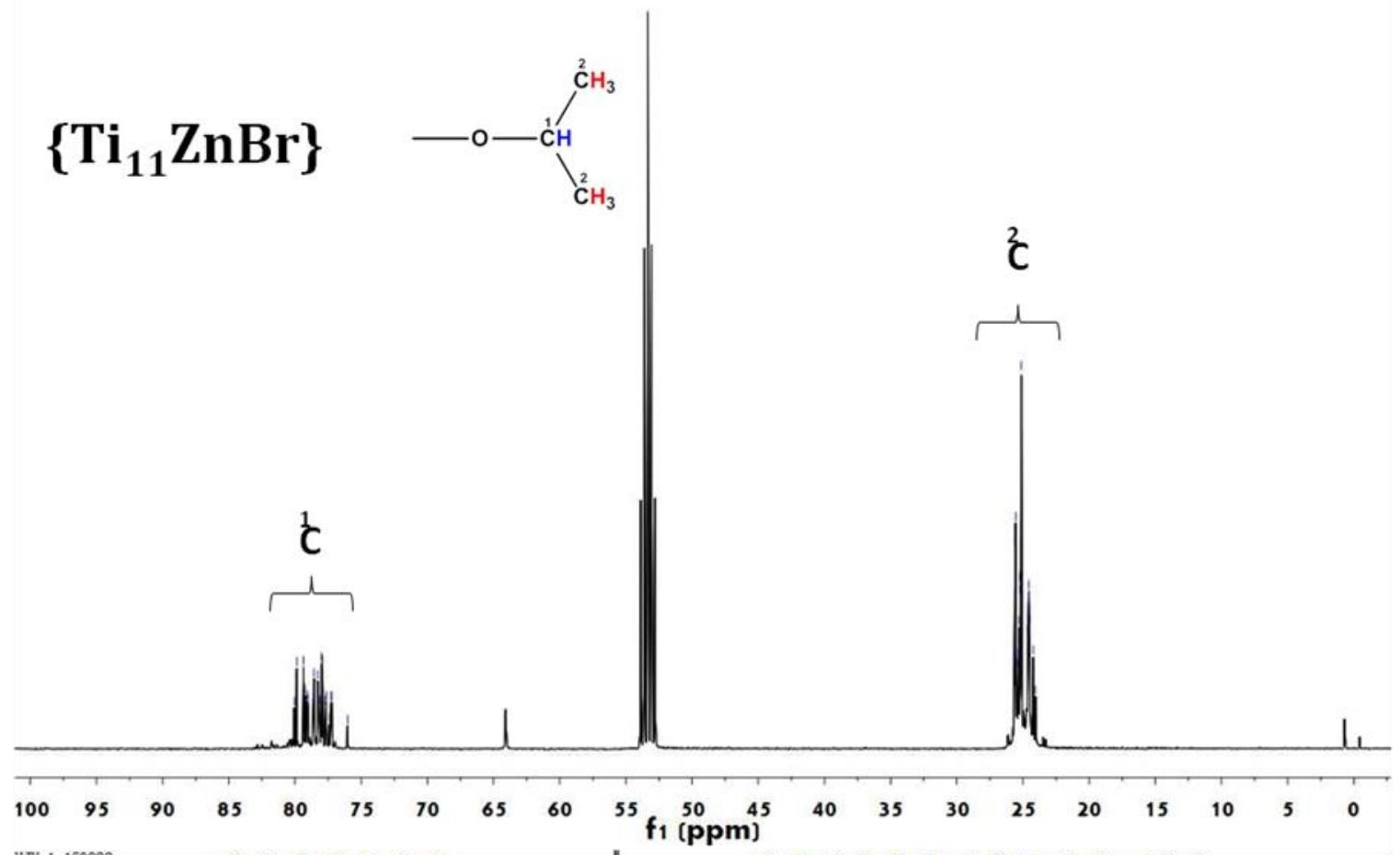

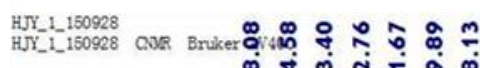

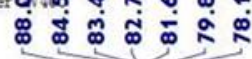

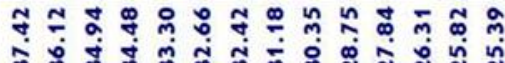

$\left\{\mathrm{Ti}_{11} \mathrm{CoCl}\right\}$

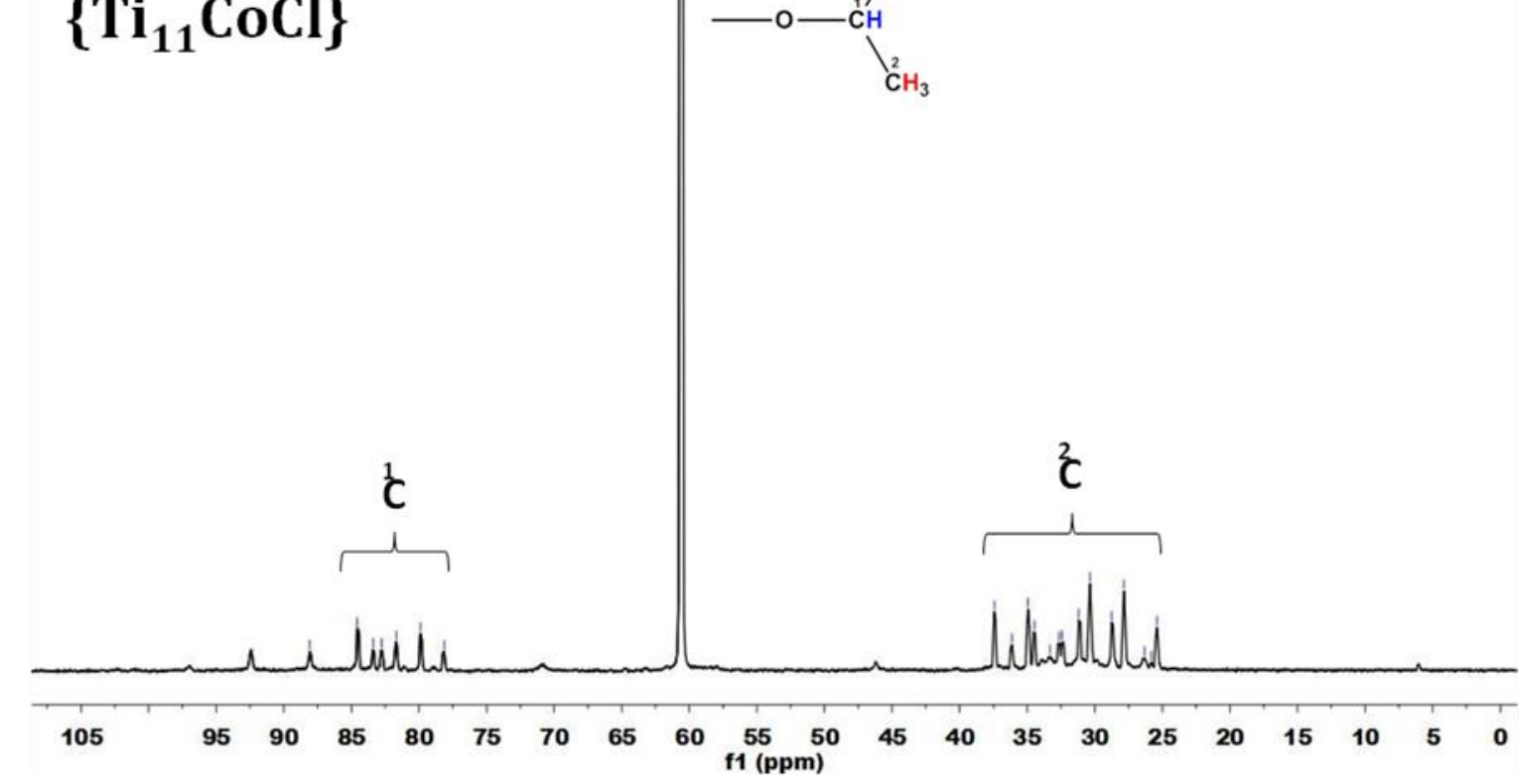




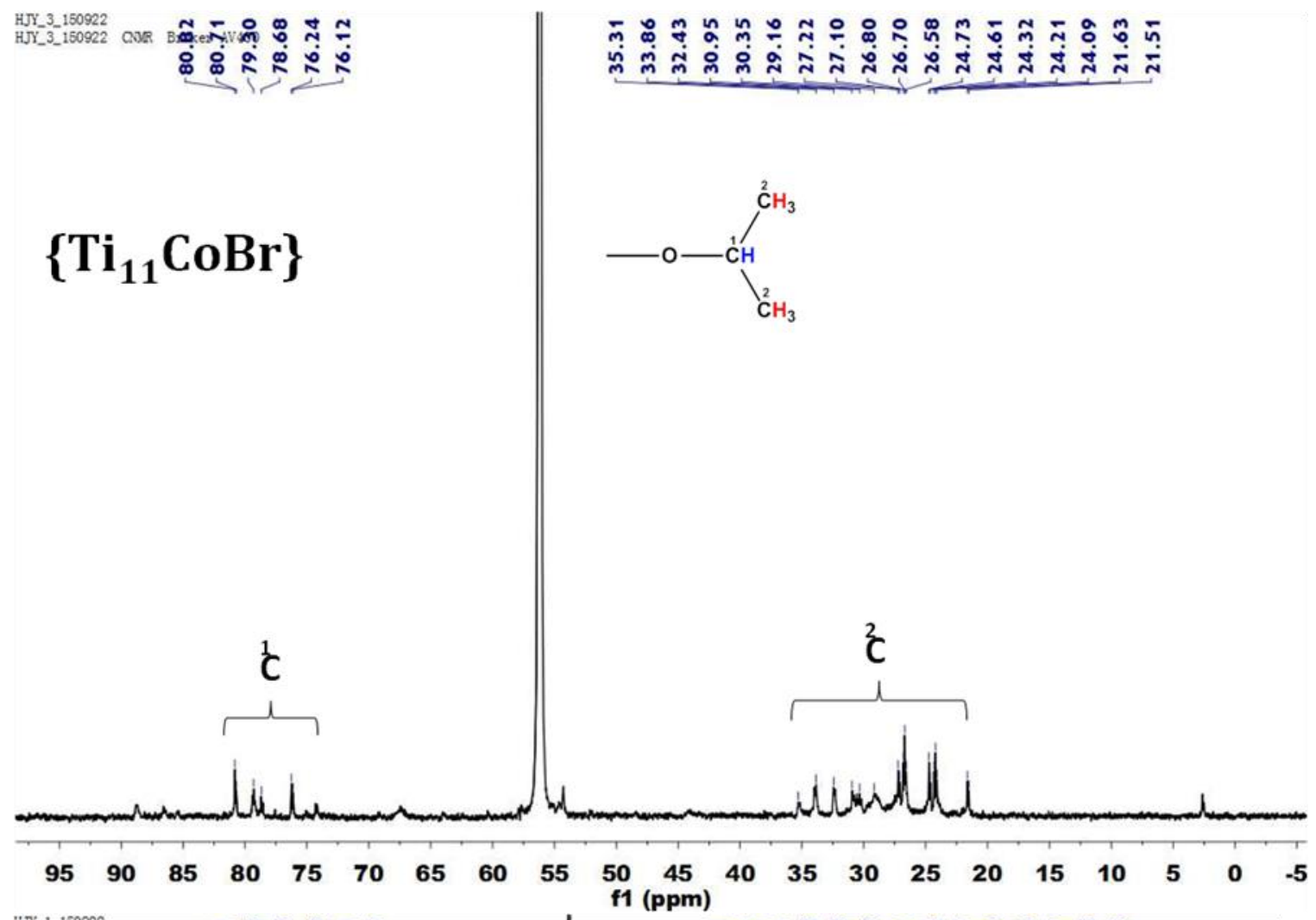

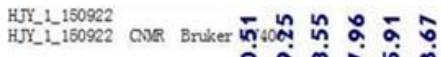

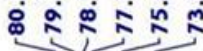

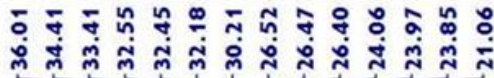

$\left\{\mathrm{Ti}_{11} \mathrm{CoI}\right\}$

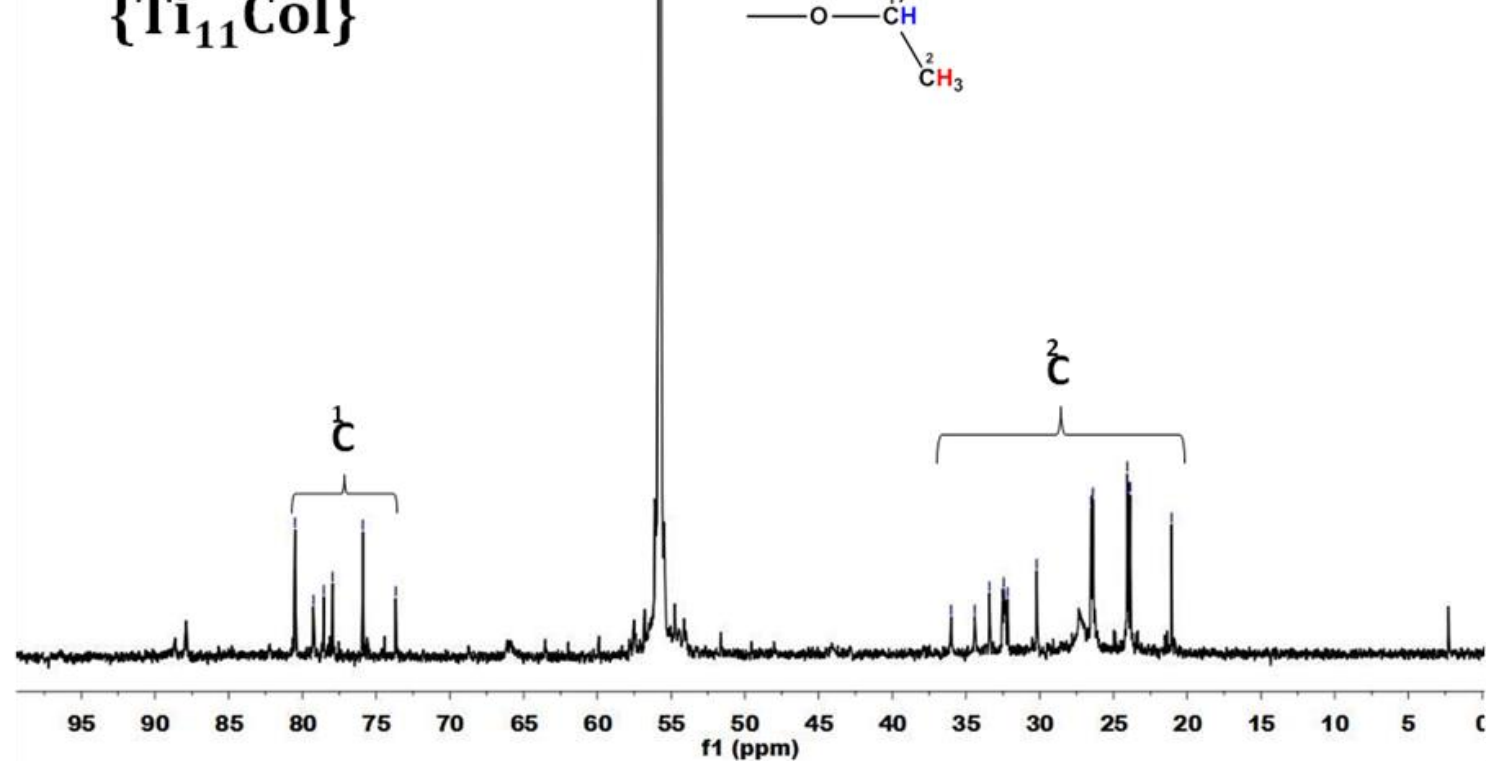



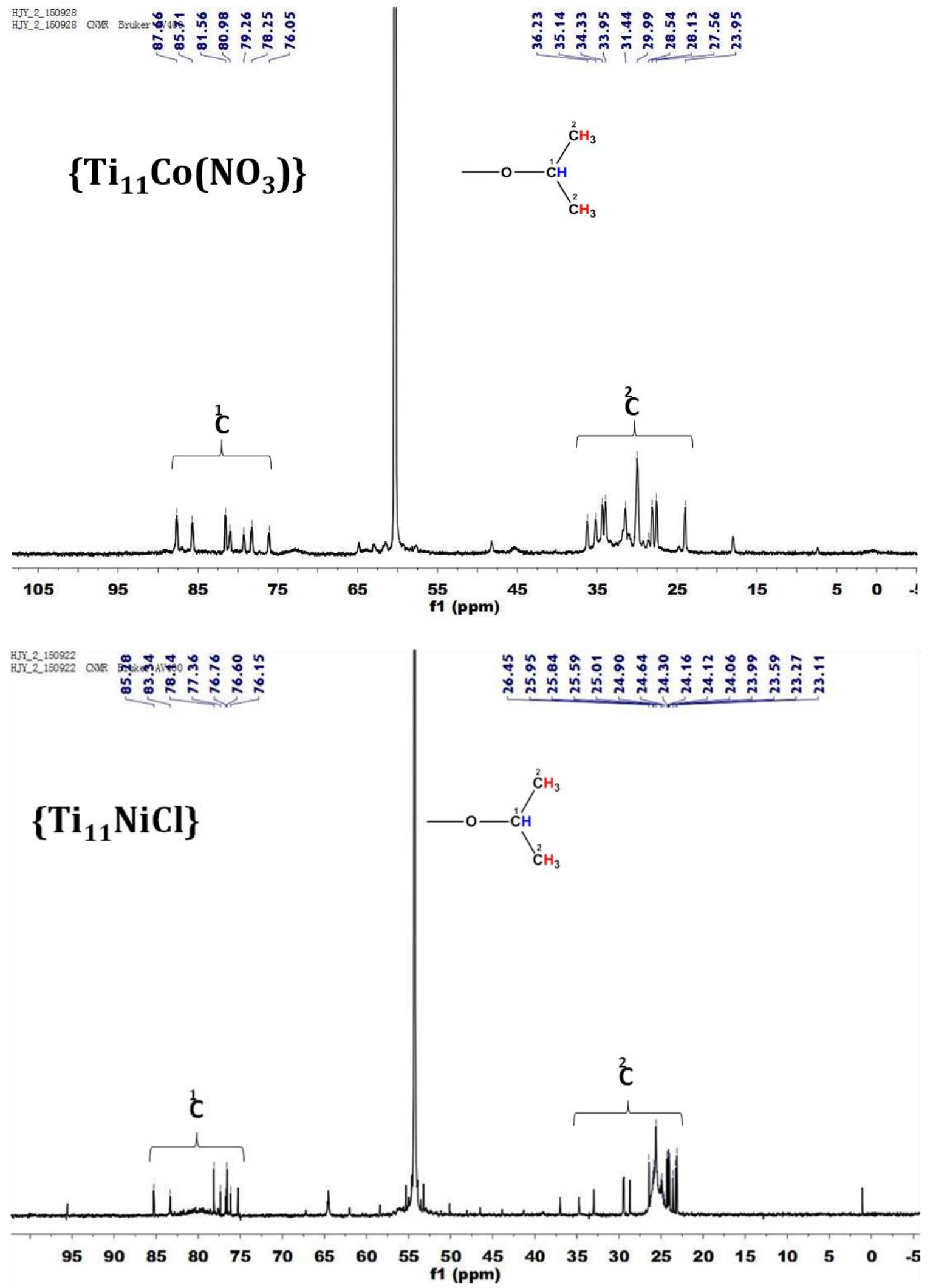


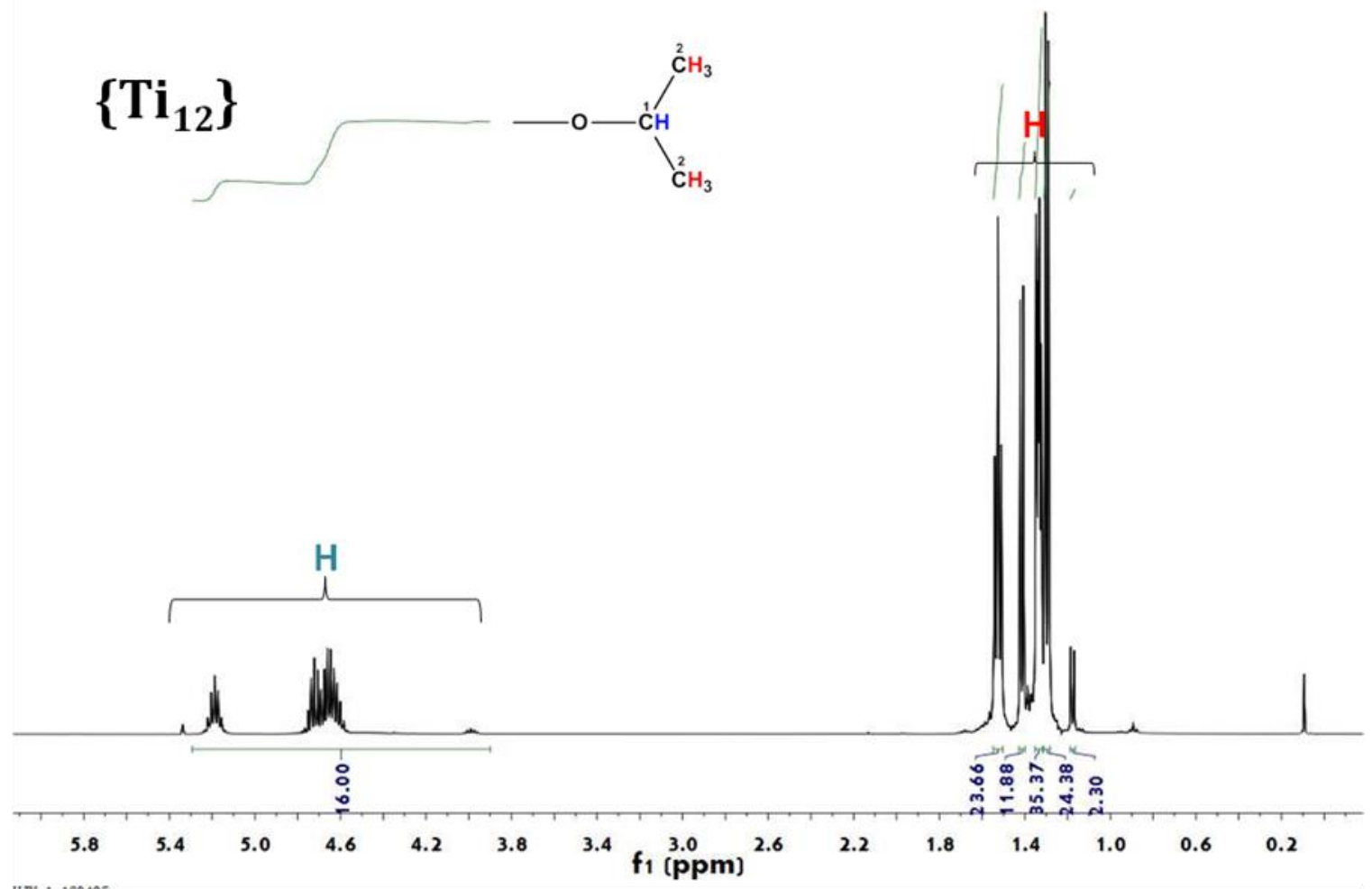

HJ__1_160405
HJY_1_160405 HNR Bruker AV400
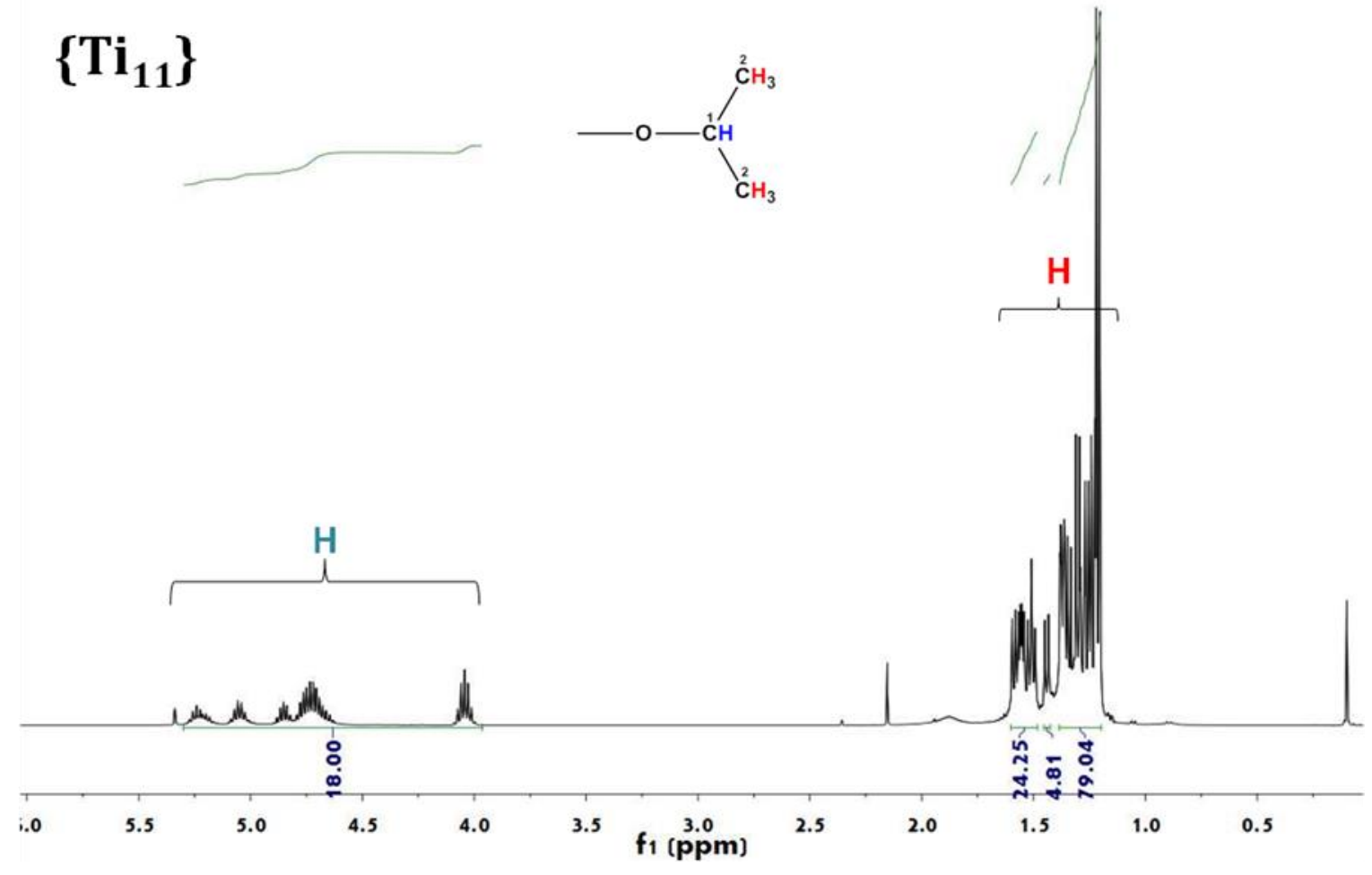

Figure S4. ${ }^{13} \mathrm{C}$ NMR and ${ }^{1} \mathrm{H}$ NMR spectra.

\section{Band gap measurements}



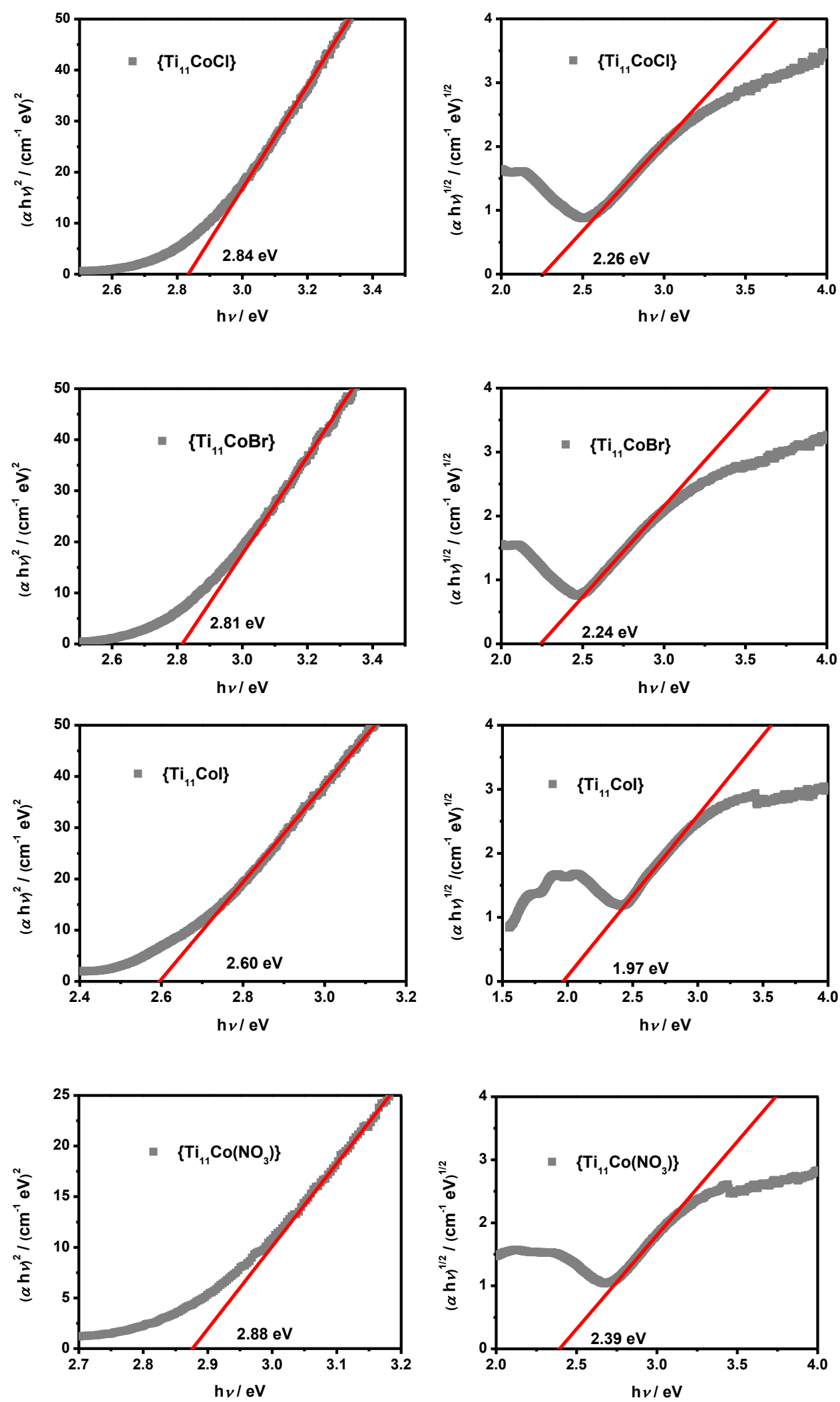

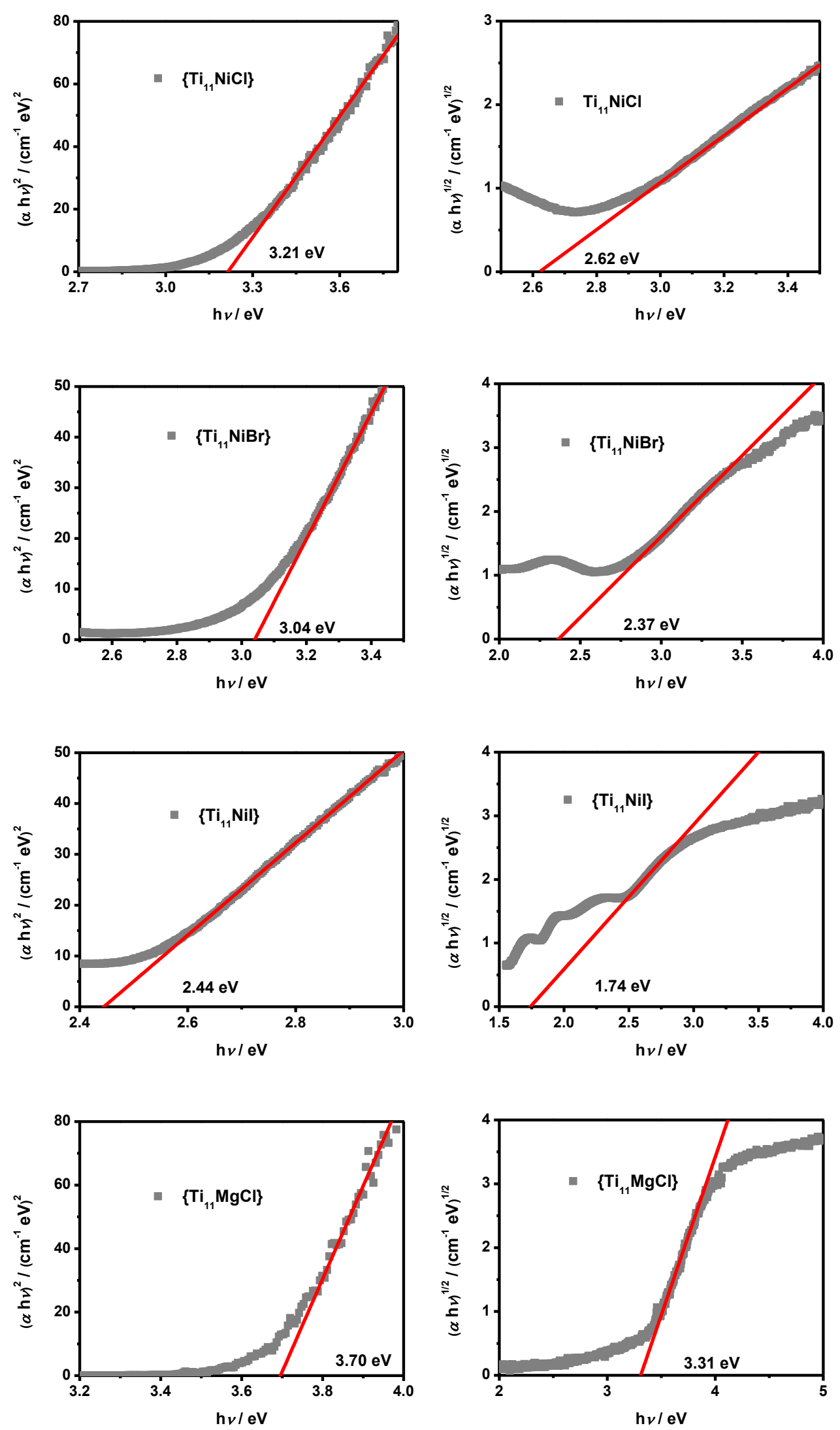

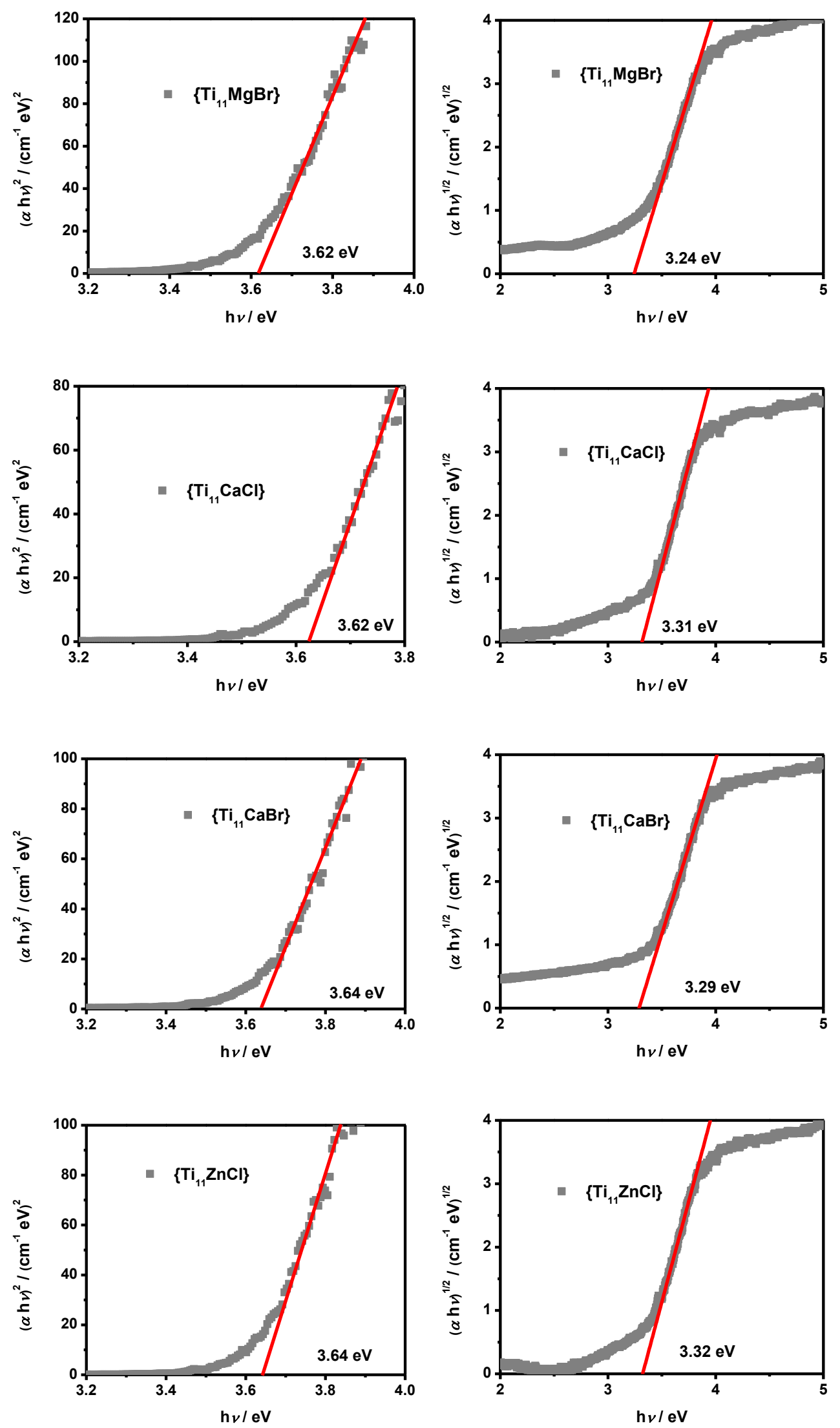

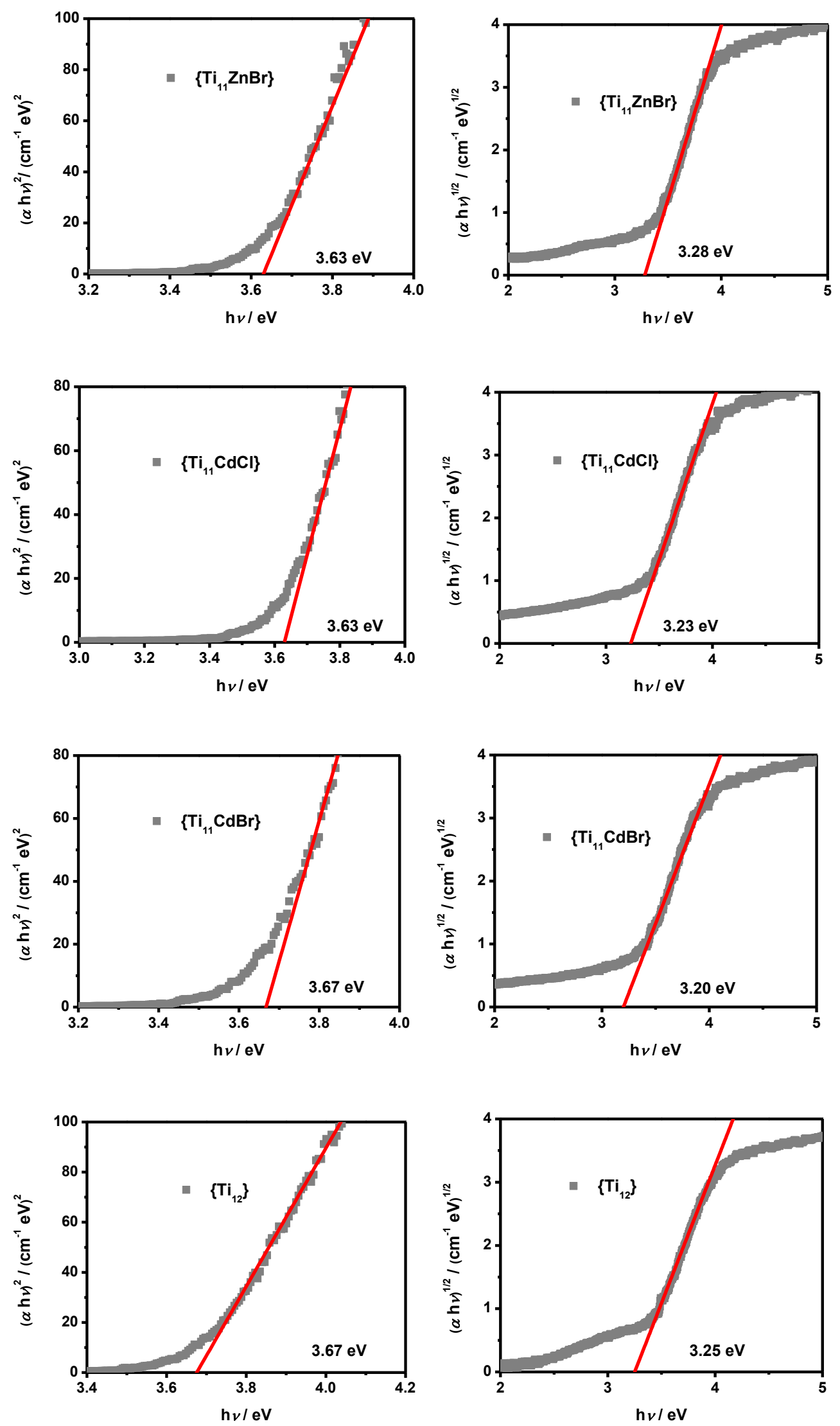

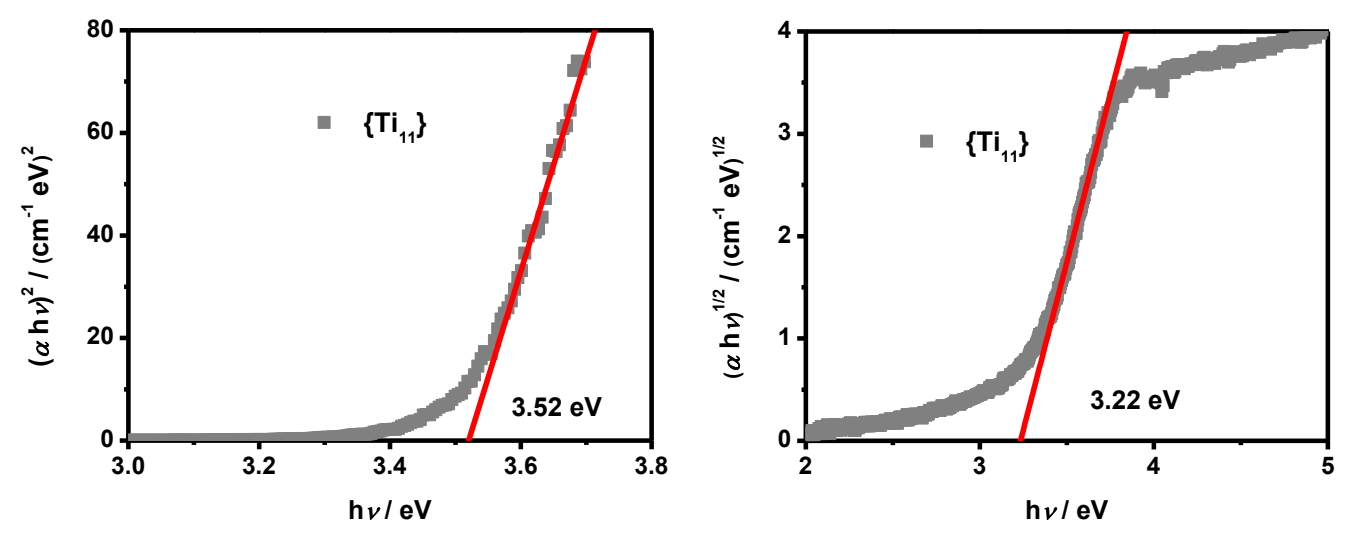

Figure S5. Calculations of direct band gap and indirect band gap values based on diffuse reflectance spectra.

\section{DFT calculations}

The projected B3LYP density of states (DOS) of $\left\{\mathrm{Ti}_{12}\right\}$ and $\left\{\mathrm{Ti}_{11} \mathrm{CoL}\right\}$ are shown below:

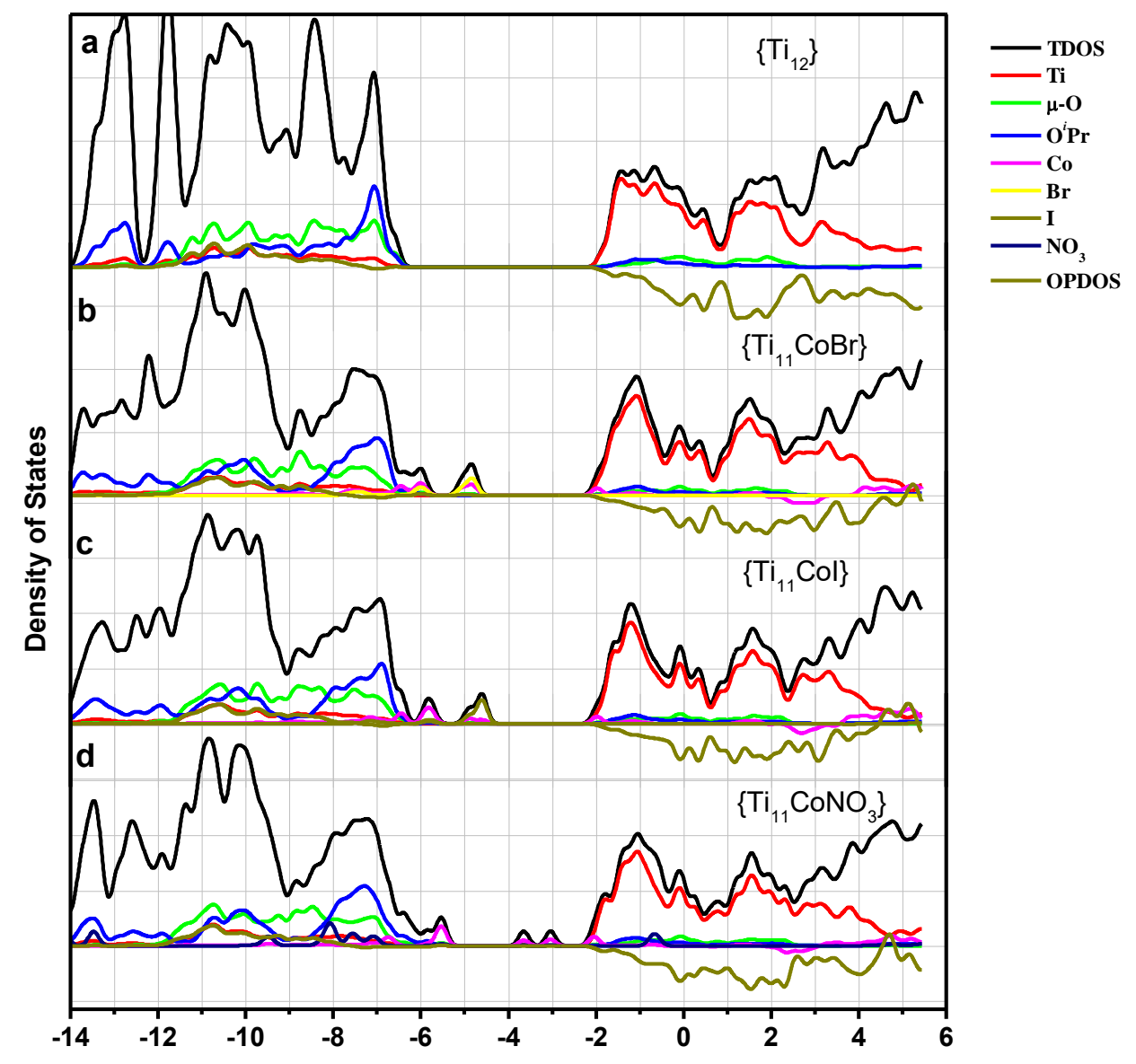

Figure S6. Density of states diagrams of (a) $\left\{\mathrm{Ti}_{12}\right\}$, (b) $\left\{\mathrm{Ti}_{11} \mathrm{CoBr}\right\}$, (c) $\left\{\mathrm{Ti}_{11} \mathrm{CoI}\right\}$ and (d) $\left\{\mathrm{Ti}_{11} \mathrm{Co}\left(\mathrm{NO}_{3}\right)\right\}$.

\section{UV-vis absorption spectra}



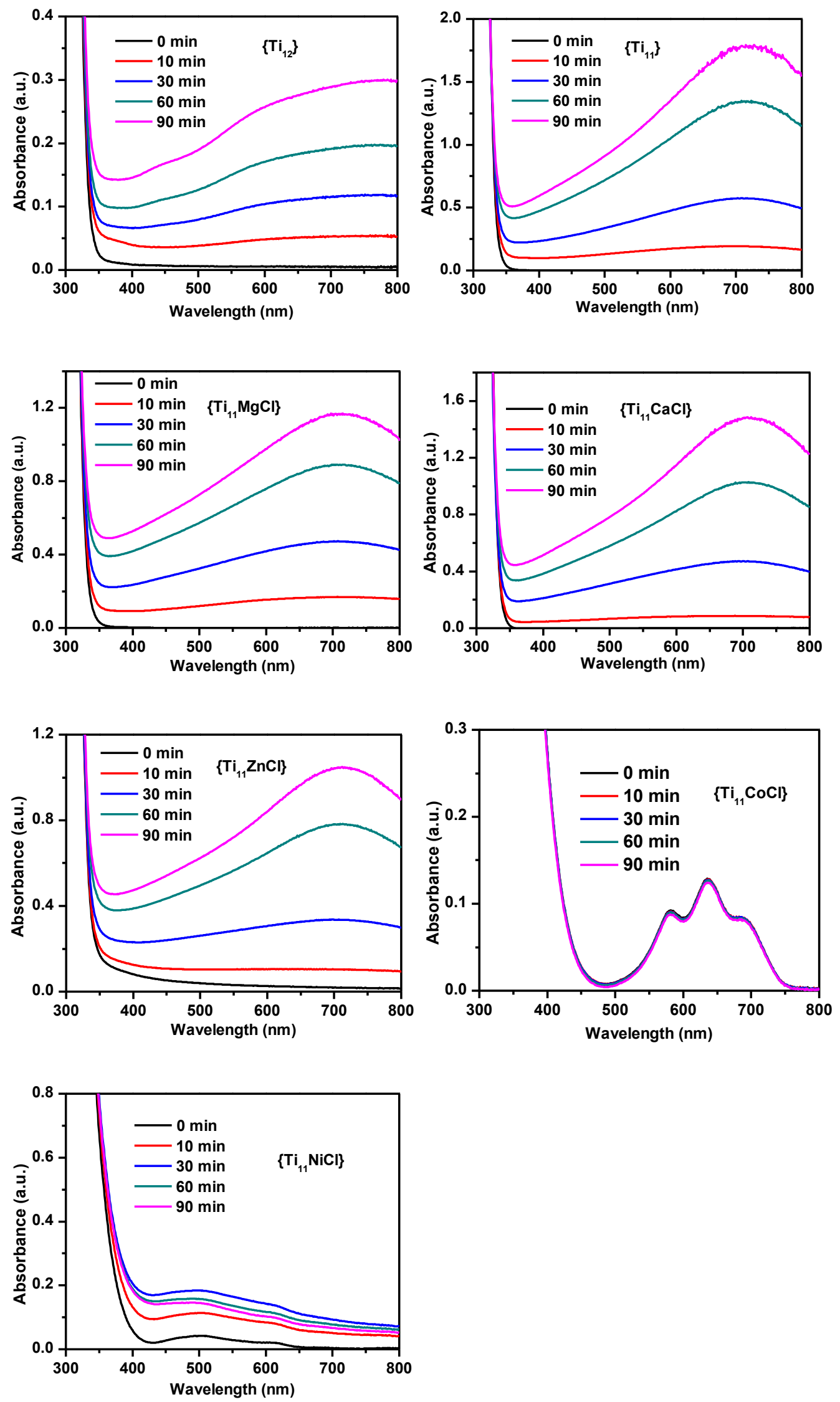

Figure S7. The UV-vis absorption spectral change of the various solutions (in isopropanol and of the same concentrations) upon illumination with UV under anaerobic conditions. 


\section{The UV-vis titrations using $\left[\mathrm{FeCp}_{2}\right] \mathrm{BF}_{4}$}

For UV-vis titration, to a cuvette containing $2.5 \mathrm{~mL}$ of the POT solution, certain amounts of $\left[\mathrm{FeCp}_{2}\right] \mathrm{BF}_{4}$ (in isopropanol) was added incrementally. This caused the absorbance of the POT solutions (with Till) to decrease. Then the absorbance at $740 \mathrm{~nm}$ was plotted against the added amounts of $\left[\mathrm{FeCp}_{2}\right] \mathrm{BF}_{4}$. The extinction coefficient of the reduced POT was then calculated according to the slope of the linear portion of the plot.
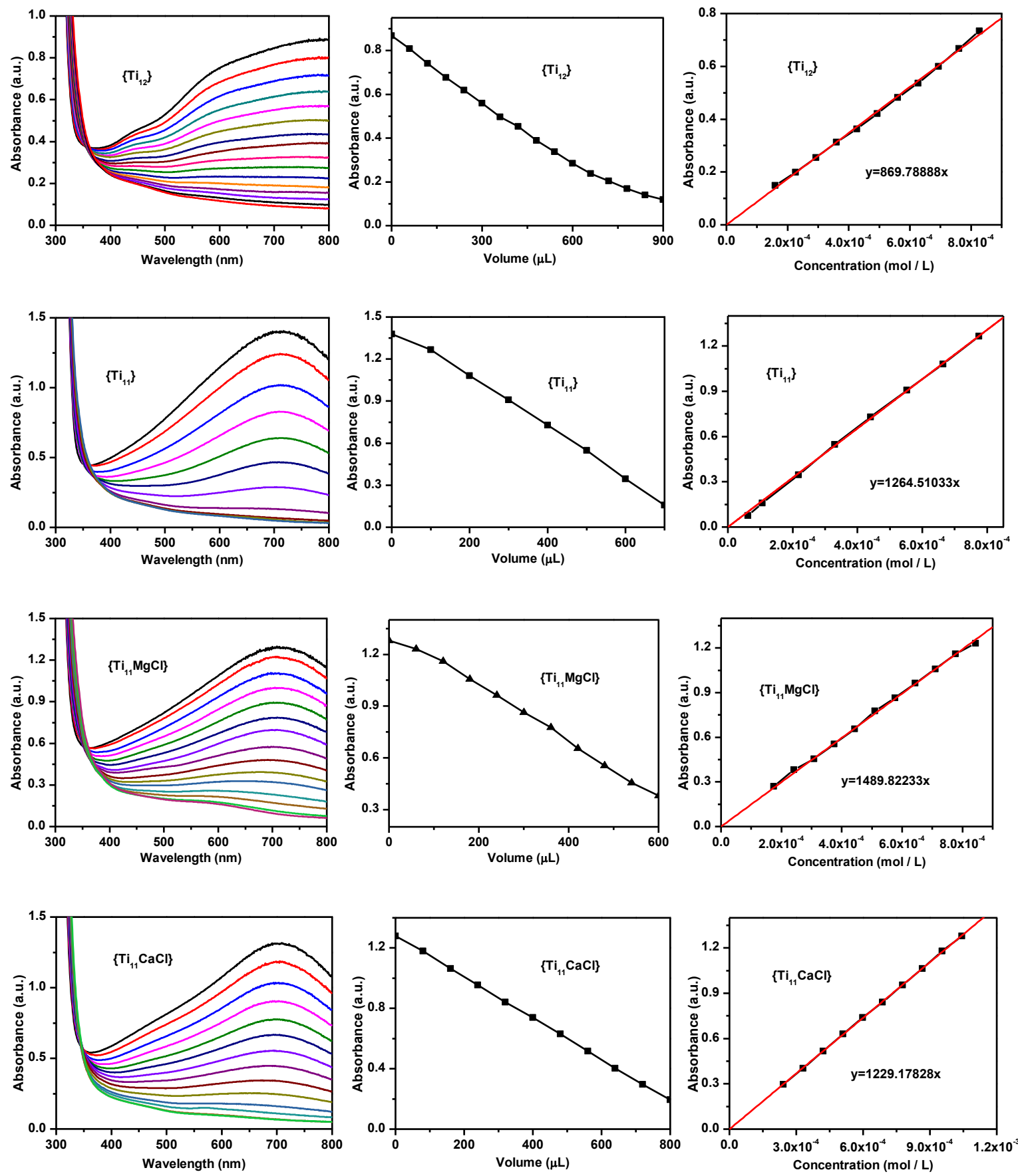

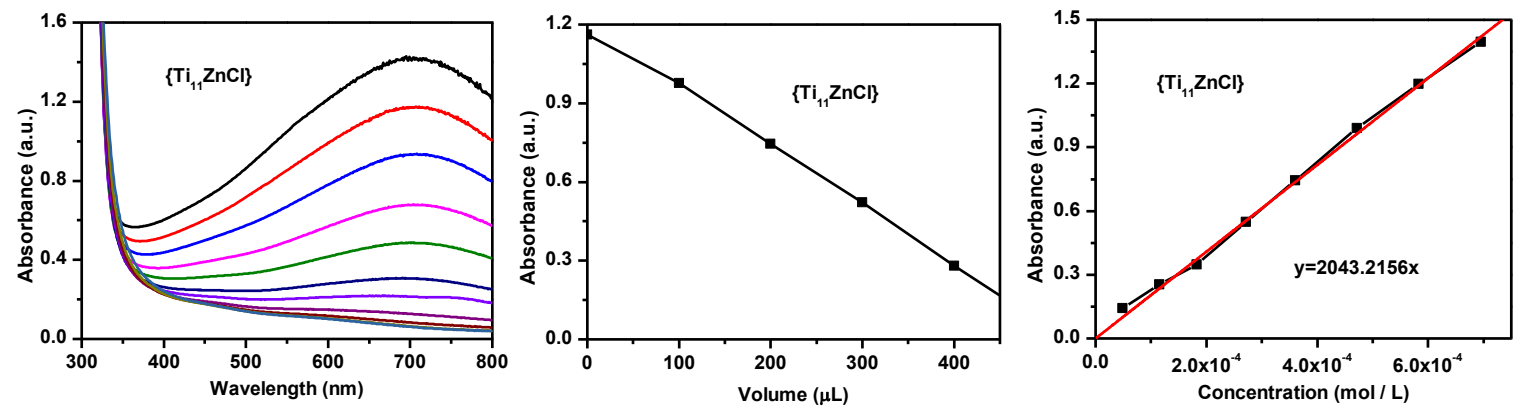

Figure S8. (Left) The UV-vis absorbance spectrum of $\left\{\mathrm{Ti}_{12}\right\},\left\{\mathrm{Ti}_{11}\right\}$ and $\left\{\mathrm{Ti}_{11} \mathrm{ML}\right\}$ titrated by $\left[\mathrm{FeCp}_{2}\right] \mathrm{BF}_{4} ;(\mathbf{m i d d l e})$ the absorbance at $740 \mathrm{~nm}$ for $\left\{\mathrm{Ti}_{12}\right\},\left\{\mathrm{Ti}_{11}\right\}$ and $\left\{\mathrm{Ti}_{11} \mathrm{ML}\right\}$ with the addition of $\left[\mathrm{FeCp}_{2}\right] \mathrm{BF}_{4} ;$ (right) the absorbance at $740 \mathrm{~nm}$ for $\left\{\mathrm{Ti}_{12}\right\},\left\{\mathrm{Ti}_{11}\right\}$ and $\left\{\mathrm{Ti}_{11} \mathrm{ML}\right\}$ as functions of the concentrations of $\mathrm{Ti} i^{\mathrm{III}}$.

\section{The ESR spectra and the simulated curves}
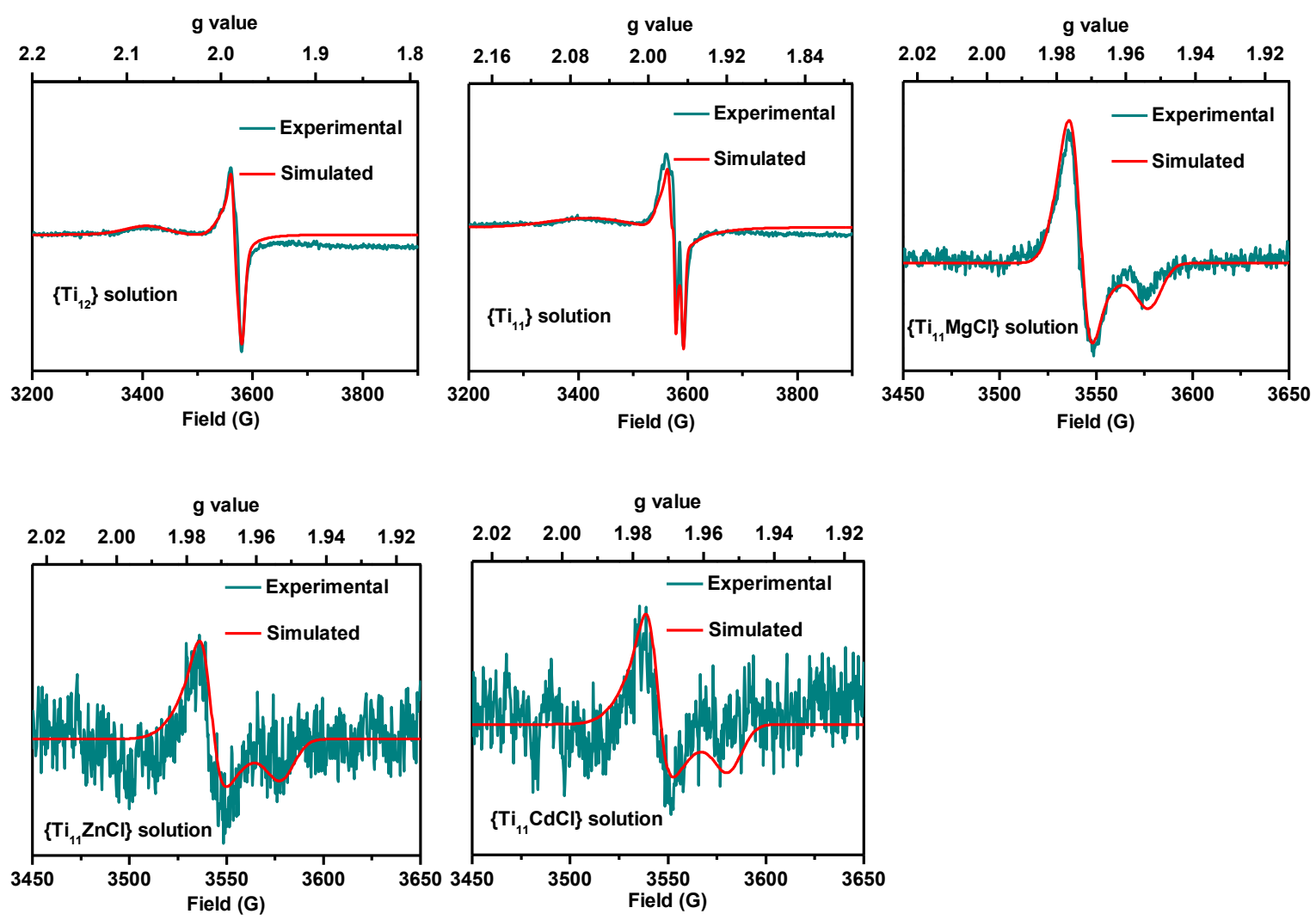

Figure S9a. The ESR spectra and the simulated curves of $\left\{\mathrm{Ti}_{12}\right\},\left\{\mathrm{Ti}_{11}\right\}$ and $\left\{\mathrm{Ti}_{11} \mathrm{ML}\right\}$ solutions in isopropanol under anaerobic conditions. 

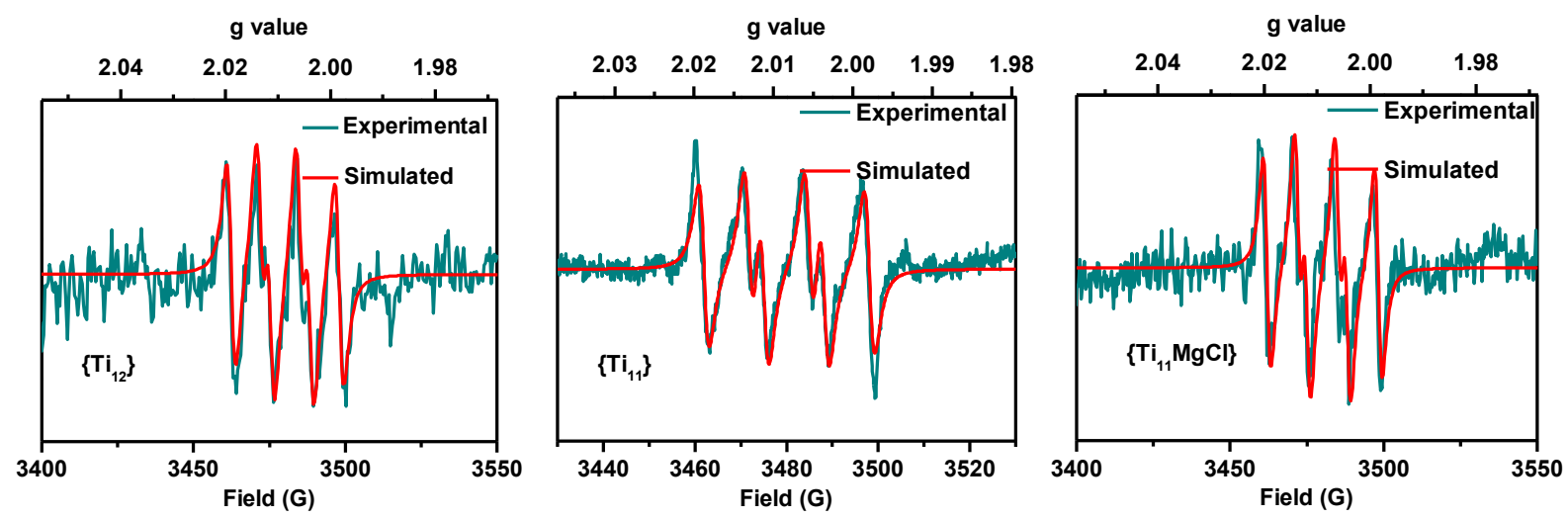

Figure S9b. The ESR spectra and simulated curves of DMPO-OOH. Conditions: isopropanol solutions and under $\mathrm{O}_{2}$.

\section{The photocurrent measurements}
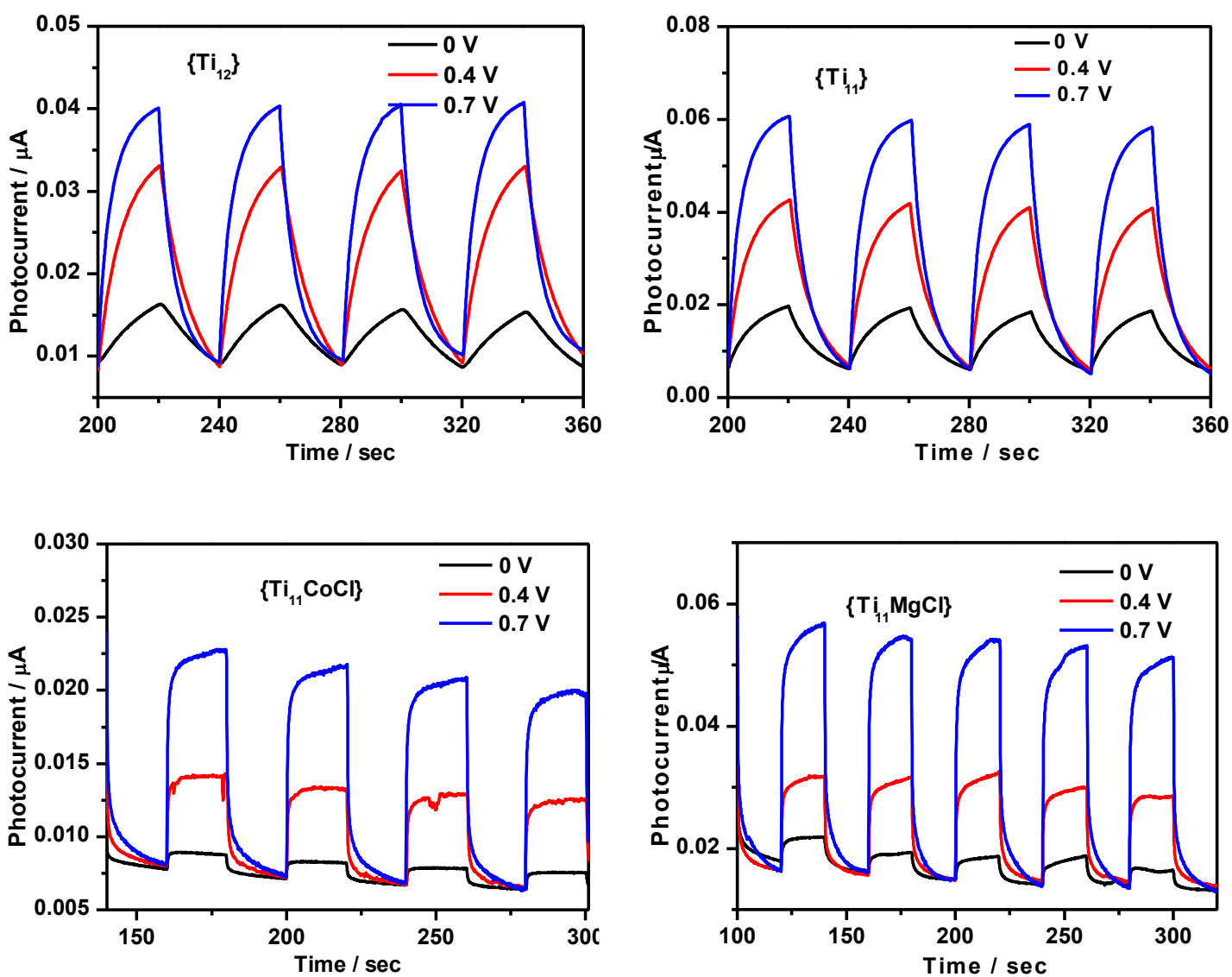

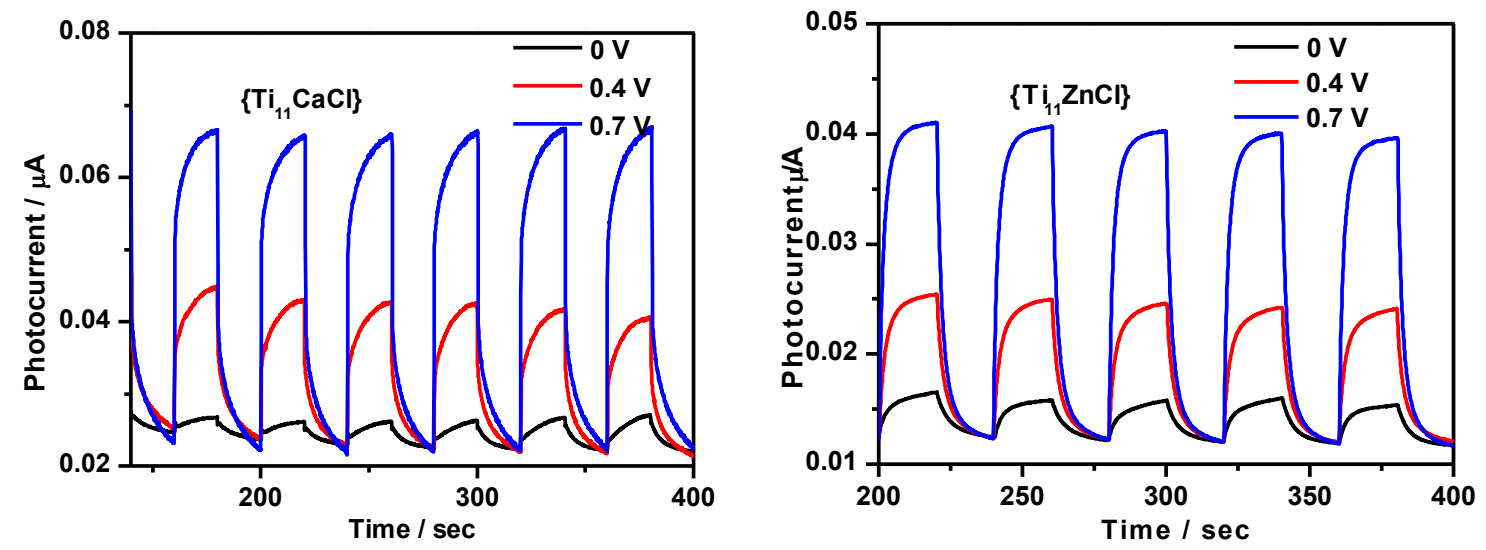

Figure S10. Time-resolved photocurrent of the $\left\{\mathrm{Ti}_{12}\right\},\left\{\mathrm{Ti}_{11}\right\}$ and $\left\{\mathrm{Ti}_{11} \mathrm{ML}\right\}$ in response to several UV on/off cycles.

\section{Photoluminescence decay spectra}

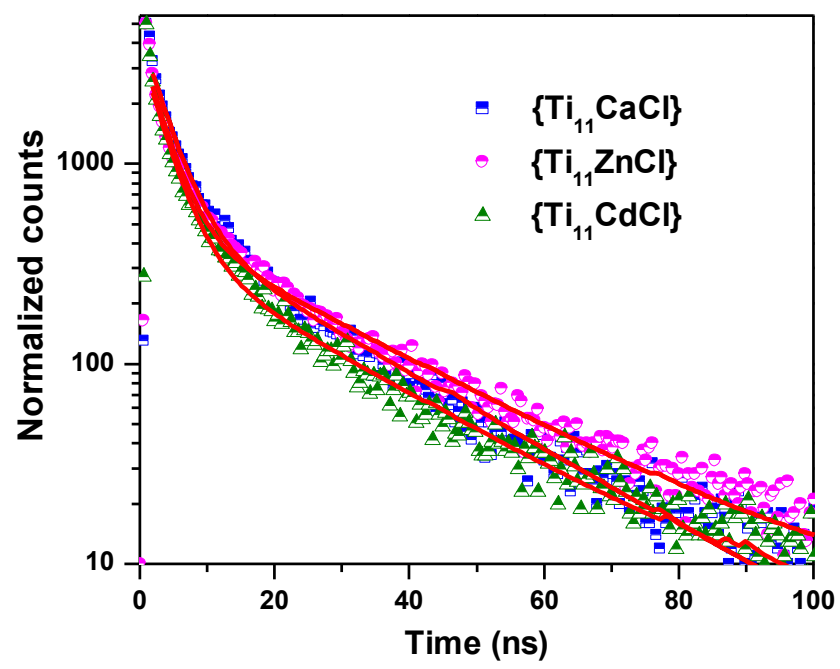

Figure S11. Photoluminescence decay spectra of $\left\{\mathrm{Ti}_{11} \mathrm{CaCl}\right\},\left\{\mathrm{Ti}_{11} \mathrm{ZnCl}\right\}$ and $\left\{\mathrm{Ti}_{11} \mathrm{CdCl}\right\}$ at $468 \mathrm{~nm}$. 